RAINER SCHMALZ-BRUNS

\title{
Gemeinwohl und Gemeinsinn im Übergang?
}

\section{Demokratietheoretische Aspekte transnationaler Integrationsprozesse}

Im klassisch-republikanischen Denken (von den Verfassungstypologien im Anschluß an Aristoteles bis zu den Zyklentheorien der klassischen Historiographie) hatte der Gemeinwohlbegriff Status eines theoretischen wie praktischen Leitbegriffs der politischen Theorie; ${ }^{1}$ eines Leitbegriffs freilich, der seine Prominenz auch der DekadenzOrientierung der politischen Analyse verdankte und dessen normativer Gehalt sich erst im Gegenlicht einer zyklischen Verfallsdiagnostik des politischen Lebens herausstellte. Es ist die enge Kopplung des Gemeinwohls an den Gemeinsinn der Bürger (an ihre Tugendhaftigkeit also), die die Konzipierung des Geschichtsverlaufs in Sinuskurven bedingt, die sich auf eine sich entspannende negative Dialektik zwischen Gemeinwohl und Gemeinsinn zurückführen läßt: Einerseits ist danach zwar das Maximum an Gemeinwohlorientierung der Bürger identisch mit dem Maximum der guten Ordnung des Gemeinwesens, andererseits erweist sich dieser Zustand aber dadurch als endemisch instabil, daß das Maximum an Ordnung im Sinne von Glückseligkeit, Gerechtigkeit, Eintracht, Frieden, Sicherheit und materieller Wohlfahrt ${ }^{2}$ regelmäßig die Tugend der Bürger unterminiert, weil sie im wohlgeordneten Zustand des Gemeinwesens aus der Anspannung ihrer ethischen Kräfte entlassen und dazu verführt werden, sich dem Wohlleben zu überlassen und eine passiv-konsumptive Haltung gegenüber dem Gemeinwesen einzunehmen. Diese Dialektik einer zyklischen Selbstdestruktion einer im Gemeinsinn der Bürger zentrierten politischen Ordnung ist das zentrale Thema des klassischen Gemeinwohldiskurses - und in dieser Gestalt wird er heute von vielen Seiten wieder aufgerufen, wenn ein Zusammenhang hergestellt wird zwischen der wachsenden wechselseitigen Entfremdung zwischen Politik und Bürger (Politikverdrossenheit) und der rapide abnehmenden Qualität der Politik (Staats- und Steuerungsversagen).

1 Vgl. Hibst 1990, Kempshall 1999.

2 Vgl. Hibst 1990, S. 70. 
Insofern kam und kommt dem Anrufen des Gemeinwohls die anmahnende Funktion $\mathrm{zu}$, die Individuen daran zu erinnern, daß sie die Vorteile wechselseitiger Kooperation in stabilen und rechtlich geordneten Gesellschaften nur einstreichen können, wenn sie den Wert von Kooperationsbeziehungen nicht nur instrumentell, sondern als eine moralische Forderung begreifen, die mit der ethischen Selbstdeutung von Personen fundamental verschränkt und von daher auch motivational durchdrungen wird, worauf die Semantik von „Gemeinsinn“ abzielt. Eine solche Leistung konnte (und kann) dem Gemeinwohl kategorial aber nur dann angesonnen werden, wenn es mehr umfaßt als eine an der transzendentalen Reflexion auf die Bedingung der Möglichkeit von Kooperation entbundene Kooperationsmoral und in einem Zug damit auch die Frage beantwortet, warum man denn überhaupt moralisch sein sollte. Genau diesen Zusammenhang hat etwa Luhmann ${ }^{3}$ im Auge, wenn er den Gemeinwohl-Topos als „Kontingenzformel“ des politischen Systems faßt, der er die paradoxe Funktion zuweist, für und durch das politische System „Limitationen zu setzen, deren Setzung nicht sofort wieder als auch anders möglich sichtbar wird" 4 , also als eine Formel, die in Gestalt einer transzendentalen Einsicht die Bedingungen der Möglichkeit politischer Thematisierung und politischen Handelns auf eine Weise benennt, „die ihrerseits nicht mehr auf die Bedingung der Möglichkeit hin aufgelöst werden kann". ${ }^{5}$ Die Schwierigkeit, die sich mit dieser Lösung des Kooperationsproblems einstellt, besteht kategorial darin, daß dem durch das Gemeinwohl und Gemeinsinn zu domestizierenden Privatinteresse auch ein Interesse am Gemeinwohl angesonnen werden muß, obwohl es doch eigentlich nur im Interesse des Gemeinwohls, nicht aber im Privatinteresse liegt, eine Unterscheidung zwischen Gemeinwohl und Privatinteresse überhaupt einzuführen. ${ }^{6}$ Diese interne Spannung läßt sich systematisch auf zweierlei Weise auflösen. Entweder man schreibt der Verfolgung des Privatinteresses eine wenn auch verborgene Tendenz ein, aufs Ganze gesehen doch dem öffentlichen Wohl zu dienen - was dann aber den Nachteil hat, daß diese Wirkung eben kontingent und nicht mit Notwendigkeit eintritt. ${ }^{7}$ Oder aber man findet eine interne Lösung der Rationalitäts- und Moralitätsproblematik des Gemeinwohls, nach der die Orientierung am Gemeinwohl aus Gründen des Gemeinwohls mit individuellen Dispositionen nun mehr nicht auf äußere Weise rational, sondern auf interne Weise ethisch verschränkt wird - dann wird man sich aber nicht mehr auf transzendentale Einsichten allein verlassen können, sondern es geht um einen moralischen Sinn, um internalisierte moralische Überzeugungen, für die eine andere Geltungsbasis als das bloß kognitive Für-Wahr-Halten von Sachverhalten, mithin eine Art Überzeugung von der Präsenz von Idealen, die uns anziehen und unser Verhalten anleiten, reklamiert werden müßte: „Conviction in the moral sense signifies being conquered, vanquished, in our active nature by an ideal end; it signifies acknowledgement of ist rightful claim over our desire and purposes ${ }^{6.8}$.

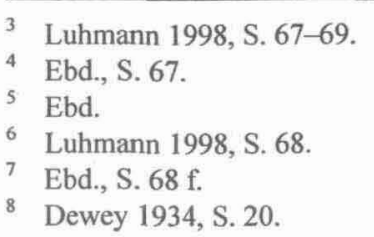


Das von Dewey ins Spiel gebrachte quasi-religiöse Moment von Werterfahrung ist dem Gemeinwohldiskurs gerade in seiner spätmittelalterlichen Blütezeit nicht äußerlich, ${ }^{9}$ und dieser transzendente Bezugspunkt scheint darüber hinaus in gewisser Weise unentbehrlich, wenn man an der Spezifik der Gemeinwohlbegriffs, der kategorialen Verklammerung von Moral, Ethik, Recht und Politik, ${ }^{10}$ festhalten möchte. Daß die damit angebotene Lösung des Stabilisierungsproblems politischer Gemeinwesen auch heute noch erfolgreich sein könnte, dagegen sprechen zunächst allerdings eine Reihe von epistemischen, kulturellen und politischen Gründen, die insgesamt eher nahelegen darauf zu verzichten, das Gemeinwohl in seiner leitbegrifflichen Stellung zu restaurieren und sich forschungspragmatisch auf die Untersuchung der liminalen, problemanzeigenden Funktion der Gemeinwohlsemantik zu konzentrieren. ${ }^{11}$ Diese Bedenken lassen sich knapp so zusammenfassen: Wie kann die nachmetaphysische, liberalpluralistische Gesellschaft, die sich nicht mehr über eine abstrakte Definition ihrer Allgemeinheit in Konkurrenz zum Individuum setzen kann und will, mit Gemeinwohlansprüchen überhaupt umgehen; denn der liberale Verfassungsstaat, der die Grenze der Verbindlichkeit und Wirksamkeit von im allgemeinen Willensbildungsprozeß ermittelten (primär prozeduralen) Normen erfährt, wird damit auf die Aktivierung von vorpolitischen Normquellen (Lebenswelten, Kulturen, Religionen, Nationen o. ä.) verwiesen, die $\mathrm{zu}$ neutralisieren er angetreten war. So gilt es denn vielen (vor allem: liberalen) Kritikern auch als ausgemacht, daß diese Tradition des politischen Denkens unter modernen Bedingungen einer funktional differenzierten, individualisierten, pluralistischen, nicht mehr über Werte integrierten Gesellschaft ihre sozialstrukturellen, gesellschaftsorganisatorischen Anhaltspunkte, ihre Relevanz und normative Dignität unwiederbringlich eingebüßt hat. So scheint dem Gemeinwohlbegriff heute praktischpolitisch der nicht leicht zu widerlegende Verdacht eines primär ideologischen Gebrauchs anzuhaften, und politisch-philosophisch bildet er bestenfalls einen partikularistischen Stachel im Fleisch der universalistisch orientierten Vernunftphilosophie in der Tradition der Aufklärung, was den Versuch einer erneuten Profilierung von vornherein mit dem Stigma gegenaufklärerischen, hinterwäldlerischen Treibens belasten könnte. Ganz in diesem Sinne markiert etwa Frankenberg (1995) an Versuchen der Reethisierung der Politik auf der Basis von ontologisch fragwürdig gewordenen „Wertverhalten $^{46}$ (Scheler), ${ }^{12}$ das Problem zunächst einer Reduktion der Idee der demokratischen Republik (gefaßt im Kern als Horizontalität der Aktivbürgerschaft in säkularisierten Gesellschaften) ${ }^{13}$ auf einen sittlich abgezweckten Staat, der wertobjektivistisch noch auf die Gesinnungen seiner Untertanen durchgreifen muß, und dem dann im Begriff der Bürgertugend ein Modell der objektiv wertbezogenen internen Kontrolle und Eindämmung von subjektiver Handlungsmächtigkeit entspricht. Was ihn daran irritiert, ist ein ethisch gestimmter Totalitarismus; deshalb gilt ihm auch als ausgemacht, daß sich

\footnotetext{
9 Vgl. Kempshall 1999, S. 24 f. u. 348-354.

10 Vgl. Hibst 1990, S. 94.

11 Vgl. Münkler/Fischer 1998, S. 8.

12 Zit. nach Wingert 1993, S. 279 f.

13 Ebd., S. 28 ff.
} 
solche Versuche einer gleichsam nachgeahmten Wertbindung nur noch aus ziemlich trüben Quellen speisen können dürften. ${ }^{14}$

Diese Bedenken richten sich freilich nur gegen mögliche Versuche einer leitbegrifflichen, kategorialen Restauration des Gemeinwohls und betreffen nicht den eher problemanzeigenden Gebrauch des Gemeinwohlkonzepts in Zusammenhängen der öffentlichen Meinungsbildung oder politischen Entscheidung, mit dem sich weniger die Erwartung der Gewährleistung gemeinwohlbezogenen Handelns als vielmehr die Hoffnung verbindet, die Orientierung an den „highest common concerns" gegenüber einer pluralistischen Interessenpolitik des ,kleinsten gemeinsamen Nenners“ auch strukturell, in Form deliberierender Öffentlichkeiten und in Gestalt geeigneter institutioneller Mechanismen, im politischen Prozeß verankern zu können ${ }^{15}-$ Strukturen, die geeignet sein könnten, die soziale, sachliche und zeitliche Selbstreflexion von im politischen Prozeß artikulierten Präferenzen anzuregen und auf diese Weise die politische Meinungs- und Willensbildung eben auch auf jene kollektiven Handlungsprobleme und Interessen zu lenken und zu fokussieren, die in dem Sinne im öffentlichen Interesse liegen, daß es sich um notwendig geteilte Interessen handelt, die sich im öffentlichen Vernunftgebrauch erschließen und die nur über kollektives Handeln befördert werden können. ${ }^{16}$ In dieser eher formalen Bestimmung wird, und das ist entscheidend, die Idee des Gemeinwohls prozedural auf die Kriterien der Inklusivität, sozialen Reflexivität und Langfristigkeit politischer Willensbildung und Entscheidungsfindung mit der Pointe hin ausgelegt, daß Politik aus einem rein voluntaristischen Verständnis von Legitimation herausgelöst wird, die sich nun in erhöhten Maße auch am kognitiven Gehalt von Interessen und Präferenzen entbindet.

In dieser Gestalt eines für kognitive Fragen offenen Konzepts der kollektiven Selbstreflexion ist das Gemeinwohl in den letzten Jahren vor allem an den Punkten wieder in das Zentrum der politikwissenschaftlichen und demokratietheoretischen Aufmerksamkeit gerückt worden, an denen auch die allgemeine Debatte sich an Problemen des Staats- und Steuerungsversagens, der Politikverdrossenheit und der abnehmenden demokratischen Qualität von Politik jenseits des Nationalstaates in der Sorge entzündet hat, daß selbst die Null-Option zur politischen Utopie werden könnte. ${ }^{17}$ Diesen zeitdiagnostischen Beobachtungen folgt denn auch ziemlich regelmäßig die therapeutische Empfehlung, sich auf Gemeinwohl und Gemeinsinn als irreduziblen Fermenten einer zivilen Praxis kollektiver Selbstbestimmung zurückzubesinnen und an diese Orientierungen für Zwecke der konstruktiven Neubeschreibung der Prozesse, Strukturen und Institutionen demokratischer Politik anzuknüpfen. In diesem Sinn ist es die Orientierung am Gemeinwohl, die dem politischen Handeln einen moralischen Rang verleiht, und der Gemeinsinn von Bürgerinnen und Bürgern wird zu einer (sogar zunehmend) unentbehrlichen, aber eher knappen Ressource politischer Legitimität, Willensbildung und Entscheidung. Das Problem, das mit den folgenden Überlegungen aufgegriffen werden soll, ist dann aber, daß vielfach angenommen wird, Gemeinsinn im anspruchs-

14 Ebd., S. 36.

15 Vgl. Goodin 1996, S. 338-341.

16 Ebd., S. 339.

17 Offe 1986. 
vollen Sinne der wechselseitig-allgemeinen Verpflichtung auf das Wohl jeweils aller anderen (Mitglieder einer politischen Gemeinschaft) könne sich nur im Horizont vorgängig gestifteter Gemeinsamkeiten und kollektiver Identitäten entfalten. Dies legt es dann nahe zu vermuten, daß auch das Gemeinwohl eine spezifische Bedeutung und einen konkreten Gehalt nur im sittlichen Horizont partikularer Gemeinschaften gewinnen kann. Genau diese Sicht wird aber nun nicht nur durch den endemischen Zug ins Universale herausgefordert, der der Idee demokratischer Selbstbestimmung eigen ist, sondern auch durch Prozesse einer zunehmenden Denationalisierung von Politik einerseits wie durch die Herausbildung regionaler oder kosmopolitischer institutioneller Ordnungen einerseits. Diese Konstellation führt uns aber vor die Alternative, ausgehend von der Prognose eines zunehmenden ethischen Substanzverlustes von Politik und einer diesen begleitenden, eskalierenden moralischen Selbstentpflichtung der politischen Subjekte, entweder auf eine Strategie der „Repatriierung “ von Politik zu setzen, oder aber den Versuch zu unternehmen, sich durch eine Neuartikulation der epistemischen Bedeutung von Gemeinwohl und der deliberativen Deutung von Gemeinsinn konzeptionell besser auf die neuen Bedingungen der postnationalen Konstellation der Politik einzustellen: Zu diesem Zweck wird in einem ersten, eher defensiven Schritt der Kritik des liberalen Nationalismus gezeigt, daß die spezifische Gemeinschaftlichkeit politischer Gemeinschaften sich weniger dem kulturellen Ferment des Nationalen als vielmehr einer reflexiven Homogenität verdankt, die in rechtsmoralischen Formen von Anerkennung gründet (2); dieses Argument wird zweitens dadurch unterstützt, daß dem Gemeinsinn auf dem Boden von Prozessen reflexiver Kooperation eine weniger ethische als mehr epistemische Bedeutung zuwächst (3), so daß schließlich das Modell deliberativer Politik eine Möglichkeit eröffnet, auch den notwendigen Übergang einer gemeinwohlorientierten Politik von der Ebene des Nationalstaates auf die Ebene transnationaler Politik konzeptionell zu plausibilisieren (4).

\section{Zur „Gemeinwohlverträglichkeit“ politischer Denationalisierung}

Wenn vielen Beobachtern politischer Prozesse in den letzten Jahren das Phänomen einer ungleichzeitigen Denationalisierung, ${ }^{18}$ also des Zurückbleibens von Prozessen einer transnationalen Institutionenpolitik hinter Prozessen der zunehmenden Denationalisierung von Problemlagen und der faktischen Internationalisierung von Problemlösungen in vielen Politikbereichen als Signum der unter dem Titel der Globalisierung zusammengefassten Entwicklungen gilt, dann markiert die sich hier immer weiter öffnende institutionelle Lücke der Politik jene Schwelle, an der eine an prozeduralen und sachlichen Gütekriterien zu messende Politik des Gemeinwohls im Übergang zur postnationalen Konstellation der Politik hängenzubleiben droht. Die Gründe dafür werden ganz allgemein darin gesehen, daß in der ,postnationalen Konstellation“ (Habermas) die ethischen Grundlagen einer gemeinwohlorientierten Politik stark strapaziert oder sogar überstrapaziert werden könnten: Durch eine soziale Überdehnung von Ver-

\footnotetext{
18 Zürn 1998a.
} 
trauen und Solidarität als den moralischen Grundlagen demokratischer Politik - so die Befürchtung - könnte es zu einer eskalierenden moralischen Selbstentpflichtung politischer Akteure und Subjekte kommen, die sich wechselseitig nur noch als Fremde wahrnehmen, gegenüber denen weder spezielle solidarische Pflichten noch die rechtlich vermittelten Formen staatsbürgerlicher Anerkennung greifen. In dieser Sicht tritt dann umgekehrt die konstitutive Bedeutung nationalstaatlich fixierter politischer Gemeinschaften für eine gemeinwohlorientierte Politik deutlich hervor, die dann als ,essentielle Regulative ${ }^{\text {c19 }}$ des binnenstaatlichen Gemeinwohls fungieren. Eben dadurch könnte man sich veranlaßt sehen, gerade im Lichte von Gemeinwohl und Gemeinsinn der „Zwischenwelt des Nationalen durchaus ein eigenes und bleibendes normatives Rationalitätsprofil ${ }^{‘ 20}$ zuzuschreiben - der Grund dafür wäre letztlich darin zu sehen, daß - mit den Worten von Rawls - es jeweils nur gruppenspezifische, partikulare Formen der Verbindung des Guten mit dem Vernünftigen geben kann, in denen sich der demokratiekonstitutive, anerkennungstheoretisch gestiftete Zusammenhang zwischen Recht und Solidarität, zwischen Selbstachtung, Selbstwertgefühl und Selbstanerkennung herstellt. $^{21}$

Hinter der demokratie-normativen wie -funktionalen Rückbesinnung auf den Nationalstaat und der entsprechenden Skepsis gegenüber großräumigen Projektionen eines kosmopolitischen Institutionalismus steht also die allgemeine Befürchtung, daß entgegen der optimistischen Vermutung, wonach neu entstehende institutionelle Räume durch nachwachsende gemeinschaftliche Bindungen durchzogen und durch neu entstehende Solidaritäten auch politisch stabilisiert würden, die durch eine Art Gesetz der Nähe bestimmte ethische Infrastruktur politischer Vergemeinschaftung allzu sehr strapaziert würde - mit der Folge einer eskalierenden moralischen Selbstentpflichtung der politischen Subjekte ${ }^{22}$ einerseits und einer strukturell abnehmenden Verpflichtungsfähigkeit des Staates ${ }^{23}$ andererseits. Diese komplementären Diagnosen sind deswegen ernstzunehmen, weil sie das Wechselverhältnis von rationalen und moralischen Motiven beleuchten, das die Belastbarkeit der ethischen Grundlagen der Demokratie bestimmt. So ist es durchaus plausibel anzunehmen, daß eine im Zuge der Internationalisierung der Wirtschaft auf negative Integration, also auf marktfördernde und -stützende Politiken umgestellte staatliche Politik sowohl gegenüber den Gewinnern wie Verlierern dieser Prozesse die Fähigkeit verliert, sie entweder in solidarische Verpflichtungen einzubinden oder auf die Akzeptanz mehrheitlich getroffener Entscheidungen zu verpflichten: Rationale Motive für solche Einstellungen lassen sich, so die eine Befürchtung, im Rahmen einer stärker voluntaristisch als obligatorisch verstandenen staatlichen Ordnung ${ }^{24}$ kaum entbinden. Und dem entspricht dann spiegelbildlich die andere Befürchtung, daß es im Zuge einer allein funktional begründeten Extension des staatlichen Ordnungsrahmens über die von traditionalen Gemeinschaftsbildungen gezogenen Gren-

19 Offe 1998 b, S. 102.

${ }^{20}$ Kersting 1998, S. 47.

${ }^{21}$ Rawls 1979, S. 479-481; Honneth 1992, S. 148-211.

${ }^{22}$ Vgl. Offe 1998b, S. 132-134.

${ }^{23}$ Vgl. Streeck 1998a, S. 35-38.

24 Ebd., S. 35. 
zen hinaus zu dem Streß einer moralischen und legitimatorischen Selbstüberforderung kommen könnte, der „dann die Vorwände für eine moralische Selbstunterforderung und [...] Rücksichtslosigkeit [liefert], der jene Dispositionen und institutionellen Arrangements leicht zum Opfer fallen können, welche die Akteure zur verantwortlichen Beachtung sozialer, zeitlicher und sachlicher Fernwirkungen ihres Handelns (und Unterlassens!) anhalten können" ${ }^{25}$ Hier fungieren nationalstaatliche Grenzen also gleichsam als Schutzwälle gegen moralische Überlastung und Selbstüberforderung, innerhalb derer sich in einem Klima von „Gewöhnung, wahrgenommener Bewährung und wachsender Vertrautheit ${ }^{\varsigma^{26}}$ Einstellungen bilden, die für die Übernahme solidarischer Lasten nicht nur einen instrumentellen (Gebrauchs)Wert besitzen, sondern darüber hinaus auch insofern einen normativen Gehalt annehmen, als sie die Einnahme einer moralischen Perspektive gegenüber allen anderen allererst ermöglichen und stützen. An dieser Wendung des diagnostischen Arguments allerdings bleiben zwei Punkte relativ unklar, die uns im Weiteren noch beschäftigen werden: Es ist nicht unmittelbar einsichtig, welchem Mechanismus sich die Entfaltung moralisch-universalistischer, auf die symmetrische Anerkennung aller anderen richtenden Einstellungen aus dem Boden ethischpartikularistischer Haltungen heraus verdanken soll - und es ist selbst für den Fall, daß das plausibel gemacht werden könnte, noch nicht gezeigt, daß gerade der Nationalstaat und nicht vielmehr andere Gruppenbeziehungen sub- oder transnationaler Natur den entsprechenden ethischen Vergemeinschaftungszusammenhang stiften oder stiften könnten. ${ }^{27}$ Es ist also für die Klärung der Frage nach der Gemeinwohlverträglichkeit von transnationaler Politik entscheidend, zunächst die allgemeinen Argumente zu prüfen, die etwa im Rahmen eines liberalen Nationalismus für die Verteidigung des besonderen ethischen Gehaltes und Wertes partikularer, gemeinschaftlicher Bindungen ins Feld geführt werden - als exemplarisch können hier die Überlegungen David Millers in seinem Buch On Nationality gelten.

Der Herausforderung eines Nachweises der demokratie-spezifischen Leistungen national gebildeter kollektiver Identitäten ist sich Miller durchaus bewußt, wenn er aus den genannten Komponenten das Motiv einer demokratie-ethischen Verteidigung der Idee der Nationalität formt. Danach liegt der grundlegende Fehler der Befürworter einer kosmopolitischen Reaktion auf die Prozesse der Globalisierung in dem Versäumnis

„to look realistically at the conditions under which the polarizing effects of the global market can be mitigated. The welfare state - and indeed, programmes to protect minority rights - have always been national projects, justified on the basis that members of a community must protect one another and guarantee one another equal respect. If national identities begin to dissolve, ordinary people will have less reason to be active citizens, and political élites will have a freer hand in dismantling those institutions that currently counteract the global market to some degree". 28

${ }^{25}$ Offe 1998 b, S. 133 f.

${ }^{26}$ Ebd., S. 132.

27 Vgl. Young 1998.

${ }^{28}$ Miller 1995, S. 187, Hervorhebungen im Original. 
Auf diese Herausforderung reagiert er mit drei Behauptungen, die das Gerüst seiner Argumentation bilden: Er behauptet erstens ein Recht auf nationale Zugehörigkeit als wesentlichem und zudem in gewisser Weise unentrinnbarem Bestandteil individueller Identität; diese individualethische Perspektive ergänzt er dann zweitens um die metaethische These, daß Nationen als ethische Gemeinschaften zu verstehen sind, die wichtige Orientierungspunkte und Markierungen auf die „ethische Landkarte" von Subjekten projizieren - woraus sich dann relativ zwanglos drittens der Anspruch nationaler Gemeinschaften auch auf politische Selbstbestimmung ergeben soll. ${ }^{29}$

An diesen drei Behauptungen hängt die gesamte Beweislast für die These, daß den durch das Moment der Nationalstaatlichkeit beschriebenen politischen Gemeinschaften - also dem rechtlich definierten Mitgliedschaftsstatus wie den durch eine gemeinsame Kultur gestifteten Bindungen - selber eine ethische Bedeutung zukommt, auf die sich eine partikularistische Rechtfertigung des Prinzips der Nationalität ${ }^{30}$ stützen können soll: Ethisch relevant ist das Prinzip der Nationalität danach zum einen deshalb, weil Individuen erst im Horizont einer hinreichend stabilen, ihnen vertrauten Kultur bedeutsame Vorstellungen eines guten Lebens ausbilden können, die durch Einbettung in einen größeren geschichtlichen Zusammenhang zugleich bewährt und gestützt werden, so daß die geteilten Vorstellungen des guten Lebens schließlich auch den Boden bilden, in dem Forderungen der Gerechtigkeit so verankert sind, daß zugemutete Opfer nicht als willkürliche Lasten, sondern als Ausdruck der je eigenen Identität verstanden werden. ${ }^{31}$ Diese rechtfertigungstheoretische Summenformel läßt allerdings zunächst noch offen, ob die Bedeutung von Nationalität primär instrumentell verstanden werden oder unmittelbar auf die inhaltliche Bestimmung dessen durchgreifen soll, was in gerechtigkeitstheoretischer Perspektive als moralisch gefordert gelten kann. Obwohl Miller ${ }^{32}$ selber einräumt, daß erst mit dem zweiten Schritt der Begründung spezieller Pflichten eine ethisch vollständige Rechtfertigung des Prinzips der Nationalität gewonnen ist, rückt er die instrumentelle Bedeutung von Nationalität so in den Mittelpunkt, daß sie eine weitgehende Eigenständigkeit annimmt - ein Schritt, der schließlich für die angestrebte Verknüpfung des demokratischen Ideals kollektiver Autonomie mit dem Prinzip der Nationalität nicht ohne Folgen bleiben kann.

Um zu plausibilisieren, daß die aus der Zugehörigkeit zu einer nationalen Gruppe erwachsenden ethischen Bindungen in besonderer Weise geeignet sind, moralisch geforderte Lasten zu übernehmen und persönliche Opfer für das Wohlergehen anderer auf sich zu nehmen, stellt Miller im wesentlichen auf den Effekt einer spezifischen Senkung des Motivationsbedarfs, also auf die Einebnung des spezifischen inhaltlichen Niveaugefälles zwischen moralischen Pflichten einerseits und der rationalen Verfolgung eigener Interessen und Zwecke andererseits ab: ${ }^{33}$ Verringert sich das Gefälle schon dadurch, daß im Falle einer ethisch gestifteten Gemeinschaft die Gruppeninteressen ohnehin zu den Zielen gehören, die Individuen im Rahmen ihrer eigenen Lebenspläne verfolgen, so

${ }^{29}$ Ebd., S. 10 f.

${ }^{30}$ Ebd., S. 65.

31 Vgl. auch Ripstein 1997, S. 209.

${ }^{32}$ Miller 1995, S. $50 \mathrm{f}$.

${ }^{33}$ Ebd., S. $66 \mathrm{f}$. 
gestattet es diese Konstellation darüber hinaus, auch dem rational motivierten Individuum eine Form „lockerer Reziprozität" anzusinnen, in der es nicht mehr um die strikte, auf zukünftige Gewinne bezogene Diskontierung eigener Leistungen (Opfer) geht, sondern die sich dadurch rechtfertigt, daß

„the act of making a contribution is not a pure loss, from the point of view of the private interests of the person making it, because he is helping to sustain a set of relationships from which he stands to benefit to some degree ${ }^{\text {*34 }}$

insofern bilden Gemeinschaften dann den „natürlichen“ Boden, auf dem formale Systeme reziproker Lastenverteilung errichtet werden können.

Selbst wenn man die zirkuläre Struktur des Arguments außer Acht läßt, die sich daraus ergibt, daß Miller die erhofften Wirkungen dem ihnen zugrunde liegenden Gemeinschaftsverständnis schon vorgibt, zieht es vier weitere Sorten von Einwänden auf sich, die insgesamt erhebliche Zweifel an dem demokratie-funktionalen und -normativen Status der Kopplungsthese begründen. So fällt in soziologischer Perspektive zunächst auf, daß̉ eine Lücke kiafft zwischen der Ebene der Gemeinschaftsbildung (etwa der Familie), an denen die ins Auge gefaßten Effekte in der Regel sowohl in empirischer wie in ethischer Hinsicht plausibilisiert werden und jener (des Nationalstaates), auf denen sie zur Geltung kommen sollen. Hier werden stillschweigend unterschiedliche Kontexte der vorpolitischen und politischen Gemeinschaftsbildung in der Erwartung zur Deckung gebracht, daß die spezifischen Ressourcen gefühlsmäßiger Nähe und moralisch-rechtlich vermittelter Anerkennung einander ergänzen und sich nicht im Gegenteil in dem Sinne wechselseitig kontaminieren, daß, soweit die Geltung von Pflichten von Gefühlen abhängig gemacht wird, dies auch zu schlechten moralischen Argumenten führt. ${ }^{35}$ Diesem Defekt versucht Miller dann zwar dadurch auszuweichen, daß er die motivationale Kraft nationaler Bindungen nicht in erster Linie an primordiale, vorpolitische Kontexte des ethischen Selbst, sondern an den in einer öffentlich-deliberativen Kultur wurzelnden, spezifisch politischen Charakter einer Nation mit der These zurückbinden möchte, ,that the obligations themselves stem from a public culture that has been shaped by political debate in the past" ${ }^{\text {‘ }}{ }^{36}$ Mit diesem argumentativen Zug kann er nun zwar dem genannten Einwand Rechnung tragen, daß der Wert von Gemeinschaftlichkeit nicht darin gründet, daß Werte gemeinschaftlich vertreten werden, sondern vielmehr darin, daß diese so vertretenen Werte auch einen intrinsisch moralischen Gehalt aufweisen ${ }^{37}$ - deshalb insistiert er darauf, daß ,the obligations we now acknowledge are not merely traditional, but will bear the imprint of the various reasons that have been offered over time in the course of these debates" ${ }^{38}$ Aber er handelt sich im Gegenzug sofort zwei neue Schwierigkeiten ein, die zum einen die Bestimmung des Verhältnisses von politisch-institutionellen und primordialen Faktoren - und damit den Status der Behauptung der Pfadabhängigkeit der institutionellen Prägung einer öffentli-

34 Ebd., S. 67.

35 Vgl. Young 1998, S. 438-441.

${ }^{36}$ Miller 1995, S. 69.

37 Vgl. McMahan 1997, S. 125-129.

${ }^{38}$ Miller 1995, S. 70. 
chen Kultur - und zum anderen das Problem betreffen, welche Rolle dem Prinzip der Nationalität in der Entscheidung der Frage zukommt, wie weit der Kreis der Personen zu ziehen ist, die sich wechselseitig Gründe für ihre Handlungen, für die Verteilung von Lasten und Vorteilen schuldig sind.

Indem Miller nunmehr die Verpflichtungskraft des Nationalen nicht mehr auf vorpolitische Loyalitäten oder den substantiellen Gehalt bestimmter Wertbezüge, sondern vor allem an eine historisch gewachsene öffentliche Argumentationspraxis und damit letztlich an eine diese Praxis stützende institutionelle Ordnung zurückbindet, muß er auch die Explikation jener ,potency of nationality as a source of personal identity [which] means that its obligations are strongly felt ${ }^{639}$ auf eine formalere Basis umstellen: Unter den denkbaren Typen spezieller Verpflichtung, die dieser Anforderung genügen und zugleich die notwendige, interne Verbindung zu jener die nationale politische Kultur prägenden Pfadabhängigkeit der Entfaltung einer spezifischen, öffentlichen Argumentationspraxis wahren, also etwa kontraktuellen, reparativen, Dankbarkeits- oder assoziativen Pflichten ${ }^{40}$ kommt den letztgenannten dann zweifellos eine herausragende Bedeutung $\mathrm{zu}$ - und das bringt Miller durch das Eingeständnis zum Ausdruck, daß er das Gravitationszentrum der ,obligations of nationality“ in das Moment der Staatlichkeit, also in ein ,formal scheme of political co-operation [...] superimposed on the national community ${ }^{* 41}$ hinein verlagert. Das ist zwar konsequent, allerdings hat diese institutionelle Wendung die insgesamt mißliche Folge, daß er nunmehr weder die Reichweite noch die spezifisch motivationale Kraft assoziativer Pflichten in den Horizont einer durch kulturelle Homogenität gestifteten, nationalen Gemeinschaftlichkeit zurückstellen kann. Ausschlaggebend für die Bestimmung von Gerechtigkeitspflichten sind in dieser Perspektive allein funktional oder institutionell induzierte Kooperationsbeziehungen, die ein Handelnder als Hintergrund seiner Aktivität annimmt. Insofern ist denn auch Young zuzustimmen, wenn sie in ihrer Kritik des liberalen Nationalismus Millers darauf insistiert, daß

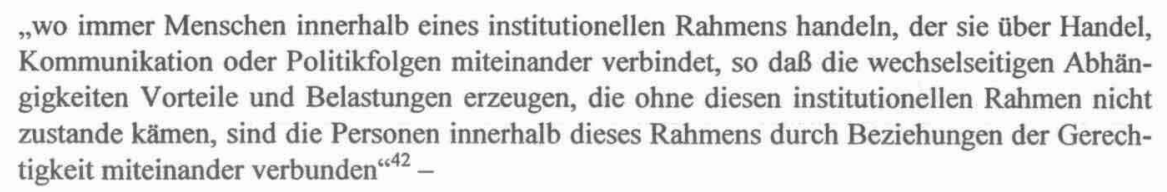

„wo immer Menschen innerhalb eines institutionellen Rahmens handeln, der sie über Handel, Kommunikation oder Politikfolgen miteinander verbindet, so daß die wechselseitigen Abhängigkeiten Vorteile und Belastungen erzeugen, die ohne diesen institutionellen Rahmen nicht zustande kämen, sind die Personen innerhalb dieses Rahmens durch Beziehungen der Gerechtigkeit miteinander verbunden ${ }^{442}$

und dieser hier von Young hervorgehobene tendenziell universalistische Zug von Gerechtigkeitspflichten macht umgekehrt darauf aufmerksam, daß die Verpflichtungsfähigkeit auch des Nationalstaates gegenüber seinen Mitgliedern dann nachlassen muß, wenn die funktionale Vollständigkeit der von ihm regulierten Interaktionsbeziehungen aufgrund der Zunahme von grenzüberschreitenden Interdependenzen immer weniger gegeben ist.

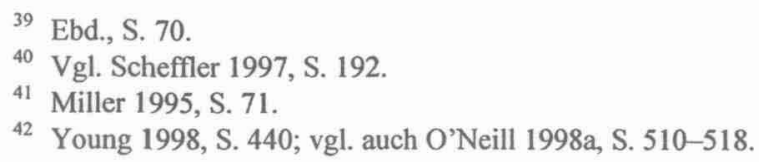


Aus diesen Gründen sieht sich schließlich auch Miller zu dem kontraintuitiven Eingeständnis genötigt, daß eine auf dem Prinzip der Nationalität fußende, eigenständige ethische Rechtfertigung des Nationalstaates nicht zu haben sein dürfte. Deshalb hat er bereits in diesem Zusammenhang den Begriff der Nation schrittweise aus Konnotationen vorpolitischer Gemeinschaftlichkeit herausgelöst und stärker in über Öffentlichkeit und staatliche Institutionen vermittelten Reziprozitätsbeziehungen verankert. In dieser in der Folge immer deutlicher hervortretenden demokratie-funktionalen Rechtfertigungsstrategie reicht die bloße Versicherung, daß ,the bonds of nationality give the practice [of co-operation, R. S.-B.] a different shape from the one it would have without it", 43 indessen nicht mehr aus. Entsprechend kehrt sich die Fragestellung um. Gefragt wird nunmehr nicht mehr danach, „whether people justifiably conceive their nationality as carrying with it a claim to political self-determination", ${ }^{44}$ sondern im Kern danach, auf welche Weise die Effektivität einer demokratischen Form der Ausübung kollektiver Autonomie $^{45}$ durch deren nationalgemeinschaftliche Einbindung gesichert werden könnte. ${ }^{46}$ Die Antwort auf diese Frage entwickelt Miller schließlich mit Bezug auf die Einsicht, daß gerade die Praxis kollektiver Selbstbestimmung ein hohes Maß an vertikalem (institutionellem) und horizontalem Vertrauen zwischen einander fremden Personen voraussetzt, unter den zwei Gesichtspunkten rationalen Vertrauens einerseits und deliberativen Vertrauens andererseits. Freilich scheitert das Argument auch dieses Mal wiederum daran, daß es ihm nicht gelingt, plausibel zu machen, daß vorgängige gemeinschaftliche Bindungen, wie er reklamiert, ${ }^{47}$ eine wichtige und irreduzible Ressource solcher Vertrauensbeziehungen darstellen: Diese Annahme scheint sogar in doppelter Hinsicht eher unplausibel, weil er zum einen von der Möglichkeit einer Endogenisierung (und dafür stehen sowohl rationales wie auch deliberatives Vertrauen) der Erzeugung von Vertrauenskapital absieht, und weil er zum anderen in funktionaler Perspektive dem Umstand nicht systematisch Rechnung trägt, daß gerade die Effektivität einer vertrauensbasierten Regulierung und Zivilisierung von Interaktionsbeziehungen unter Bedingungen wachsender funktionaler Interdependenz auf Formen des Vertrauens angewiesen ist, die eine größere Elastizität und Plastizität aufweisen können müssen als jene, die sich allein dem (gemeinschaftlichen) Gesetz der Nähe verdanken.

Die unzureichende konzeptionelle Verklammerung der Bedarfsdiagnose mit einer genetischen (oder: Ressourcen)Perspektive zeigt sich schon im Blick auf das Phänomen rational motivierten Vertrauens, das eine vertikale und eine horizontale Dimension hat. In der vertikalen Vertrauensdimension (Vertrauen zu Eliten, Institutionen) geht es u. a. um das Problem, unter welchen Bedingungen man erwarten kann, daß einseitig erbrachte Vorleistungen des Vertrauensgebers dadurch als rational gerechtfertigt angesehen werden können, daß sie tatsächlich zukünftige Ausgleichszahlungen durch den Vertrauensnehmer motivieren. Besonders riskant wird ein solches Reziprozitätskalkül dann, wenn sich die erwartete reziprozierende Handlung (wie etwa im Fall der Rentenpolitik)

\footnotetext{
${ }^{43}$ Miller 1995, S. 71.

${ }^{44}$ Ebd., S. 83.

45 Ebd., S. 89.

46 Ebd., S. 90.

47 Ebd., S. 92.
} 
so weit in die Zukunft verschiebt, daß nicht aktuell, sondern zukünftig handelnde Personen durch die Vertrauensleistung gebunden werden sollen - in diesem Fall ruht rationales Vertrauen auf normativen Erwartungen an die moralische Verläßlichkeit von Institutionen. ${ }^{48}$ In der horizontalen Dimension (als Beispiel kann hier die Implementation umweltpolitischer Programme dienen) dreht sich das Vertrauenskalkül um die Frage, unter welchen Voraussetzungen meine investiven Vorleistungen von allen anderen als ein Vertrauenssignal verstanden wird, das sie zu Handlungen veranlaßt, die zu meinem oder unserem Wohlergehen beitragen und jedenfalls die absichtliche Schädigung meiner oder unserer Interessen vermeiden.

In beiden Fällen zeigt sich nun, daß wir es im Fall von (zumindest politischem) Vertrauen mit einer dreistelligen Relation zu tun haben, in der sich Vertrauensbeziehungen zwischen einander fremden Personen nur unter Bezug auf eine gemeinsame, eine katalytische Wirkung entfaltende Norm ausbilden. Dies ist denn auch für Miller der Anlaß, die über partikulare Gemeinschaften vermittelten, tradierten und historisch bewährten Wertorientierungen in diese Funktion einzurücken, weil die an besonderen Mitgliedschaftsmerkmalen anhaftenden „kategorialen" Vertrauenserwartungen insofern als verläßlich gelten können, als „vertrauenswürdige“ Personen von außen gleichsam leicht zu erkennen sind und die Vertrauenswürdigkeit signalisierenden Merkmale weder willkürlich erworben noch einfach abgestreift werden können. ${ }^{49}$ Vor diesem Hintergrund scheint das Argument rationalen Vertrauens durchaus geeignet, die These des demokratie-funktionalen Werts nationaler Gemeinschaften zu stützen. Allerdings sind mit Blick auf die an den Beispielen sichtbar gewordenen Anforderungen an rationales Vertrauen die Schwächen dieser Konzeptualisierung schnell freigelegt: Nationale Identitäten bieten, für sich genommen, sicher noch keine verläßlichen normativen Anhaltspunkte resp. Erwartungswerte für kollektive Einstellungen etwa zu Fragen sozialer Gerechtigkeit sie sind, wie Miller selbst mit Blick auf die amerikanische Identität hervorhebt, in diesem Sinne normativ unterbestimmt, so daß man zumindest zwischen der Stärke nationaler Gefühle und ihrem spezifischen Gehalt unterscheiden müßte ${ }^{50}$ sie müssen deshalb zweitens für Zwecke eines rationalen Vertrauenskalküls auch kognitiv unterbestimmt bleiben, weil wir aus der Tatsache allein, daß es sich bei der relevanten Bezugsgruppe u. a. auch um Amerikaner handelt, noch keine starken Schlüsse ziehen können; und drittens bleiben im relevanten Sinne verhaltensprägende Wirkungen kollektiver Identitäten von ihrer Reichweite her selbst im günstigsten Fall wahrscheinlich eher auf subnationale Gruppenbildungen beschränkt - deshalb korreliert ihre vertrauensstiftende Wirkung innerhalb der eigenen Gruppe häufig mit einem verstärkten und unter Kooperationsgesichtspunkten dysfunktionalen Mißtrauen gegenüber allen anderen. ${ }^{51}$

\footnotetext{
${ }^{48}$ Auch in diese vertikale Dimension ist eine horizontale insofern eingeschlossen, als das Vertrauen in die moralische Qualität von Institutionen (vgl. Offe 1999, S. 70-76) das Vertrauen impliziert, daß auch zukünftig alle anderen in ihren Handlungen hinreichend von dieser Qualität motiviert werden.

49 Vgl. Offe 1999, S. 63 f.

${ }^{50}$ Miller 1995, S. 94.

51 Vgl. Offe 1999, S. 65.
} 
Die Problematik einer externalistischen Konzeptualisierung von politischem Vertrauen wird noch deutlicher, wenn man sie, wie Miller, ${ }^{52}$ auch auf das Phänomen deliberativen Vertrauens erstreckt. Die Leistungen, die er in diesem Zusammenhang einem national gestifteten Grundvertrauen ansinnt, bestehen in der Disposition zur reflexiven (Selbst)Evaluation der je eigenen Interessen in sozialer und sachlicher Hinsicht, so daß gewährleistet werden kann, ,that citizens [are] willing to moderate their claims in the hope that they can find common ground on which policy decisions can be based". 53 Allerdings gehen vertrauensstiftende Signale gerade im Fall von öffentlicher Deliberation weniger von kategorialen Merkmalen einer Gruppe, sondern vielmehr von der Aufrichtigkeit und Konsistenz öffentlich vertretener Argumente aus: Vertrauensstiftend wirkt hier in erster Linie die Tatsache, daß Personen im Sinne ,ziviler Integrität ${ }^{454}$ sich dadurch bewähren und als vertrauenswürdig erweisen, daß sie ihre Interessen und Meinungen mit öffentlich kritisierbaren Geltungsansprüchen vertreten.

Zusammengenommen spricht also einiges dafür, daß die Bedeutung des Nationalstaates als nicht überschreitbarer Horizont einer ethischen Vergemeinschaftung, innerhalb derer allein belastbare kooperations- und solidargemeinschaftliche Orientierungen entstehen und stabil reproduziert werden können, sowohl in funktionaler wie auch in normativer Hinsicht eher überschätzt wird. Wenn man im Gegenzug die Elemente analytisch isoliert, über die sich Prozesse politischer Vergemeinschaftung vollziehen, wie u. a. die rechtlich vermittelte wechselseitige Anerkennung von Individuen als Freie und Gleiche, die institutionell vermittelten Moralitäts- und Rationalitätsbedingungen horizontalen, zivilen Vertrauens und die über öffentliche Diskurse vermittelte soziale, sachliche und zeitliche Reflexivität der Präferenzbildung und Interessenartikulation, ${ }^{55}$ dann spricht zunächst nichts gegen die Erwartung, daß sich diese gemeinschaftsstiftenden Praktiken auch den Prozessen einer zunehmend sich denationalisierenden Politik eingeschrieben werden könnten. Kurz: Gemeinsinn als soziomoralische Ressource ist kein exogen erzeugtes Gut, daß sich durch Gebrauch und Inanspruchnahme verringert, sondern ein Gut, daß aus dem Boden sozialer und politischer Praktiken herauswächst und durch aktiven Gebrauch auch gemehrt werden kann - freilich nimmt die Orientierung am Gemeinwohl als Gütemerkmal von Politik dann auch einen eher kognitiven Gehalt an, weil die Praktiken, über die sich diese Orientierung reproduziert, selber um ein stärker epistemisches Verständnis der Legitimitätsbedingungen demokratischer Politik zentriert sind.

\section{Zu einem epistemischen Verständnis des Gemeinwohls}

Die bisherigen Ausführungen zu den Bedingungen, unter denen sich auch unter Voraussetzung einer postnationalen Konstellation die Gemeinwohlorientierung politischer Prozesse reproduzieren könnte, sind insofern defensiver Natur, als sie lediglich versuchen, die im Rahmen eines (ethischen) Ressourcenmodells vorgenommene begriffliche

\footnotetext{
${ }^{52}$ Miller 1995, S. 96-98.

53 Ebd., S. 97.

${ }^{54}$ Gutmann/Thompson 1996, S. 81 f.

55 Vgl. auch Zürn 1998b, S. 12-15.
} 
Verklammerung von Gemeinwohl und nationalstaatlich strukturierter Politik abzuweisen versuchen. Soll darüber hinaus in konstruktiver Perspektive auf legitimitätsstiftende Strukturen einer transnationalen Politik vorgegriffen werden, und soll in diesem $\mathrm{Zu}-$ sammenhang darauf abgestellt werden, die strukturbestimmenden Aspekte der Gemeinwohlidee genauer zu fassen, dann besteht die Aufgabe darin, den epistemischen Zug, der der politischen Willensbildung und Entscheidung über die Gemeinwohlorientierung auch eingeschrieben werden soll, deutlicher hervortreten zu lassen und zum konzeptionellen Angelpunkt der weiteren Überlegungen zu machen. Ein solcher Versuch zehrt offensichtlich von der Idee deliberativer Politik, die deswegen einen besonders geeigneten konzeptuellen Rahmen abgibt, weil ,a discursive or communicative model of democracy is particularly conducive to international society because, unlike other models of democracy, it can downplay the problem of boundaries" ${ }^{\text {“ }}{ }^{56}$ Verantwortlich für diesen Effekt ist nicht zuletzt die dadurch eröffnete Möglichkeit, die Demokratie unmittelbar im spezifisch diskursiven Charakter politischer Interaktionen zu lokalisieren und sie deshalb nicht mit einem zu stark ethisch gefaßten Grundverständnis zu belasten. Dieser Zug wiederum gründet normativ gesehen zum einen in der mit der Idee des öffentlichen Vernunftgebrauchs vorgenommenen Erweiterung des Verständnisses von Freiheit und Gleichheit insofern, als nunmehr zum einen die strengen Reziprozitätsbedingungen einer öffentlichen Argumentationspraxis die wechselseitige Anerkennung von Beteiligten als Freie und Gleiche vermitteln, ${ }^{57}$ und zum anderen in der ,epistemischen" Ergänzung der voluntaristischen Auffassung individueller Freiheit und kollektiver Selbstbestimmung (im Sinne der Chance, in der Verfolgung primär privater Interessen die damit verbundenen Präferenzen wirksam zur Geltung zu bringen): Dann nämlich, so Habermas,

„zieht das demokratische Verfahren seine legitimierende Kraft nicht mehr nur, und nicht einmal in erster Linie, aus Partizipation und Willensäußerung, sondern aus der allgemeinen $\mathrm{Zu}$ gänglichkeit eines deliberativen Prozesses, dessen Beschaffenheit die Erwartung auf rational akzeptable Ergebnisse begründet"، ${ }^{58}$

In dieser Formulierung sind die wesentlichen Elemente einer vorläufigen Definition deliberativer Politik bereits enthalten.

Entscheidend ist, daß die verbreitete Vorstellung einer Reduktion von Politik und politischen Entscheidungen auf instrumentelle und strategische Rationalität zurückgewiesen und damit gegenüber aggregativen Modellen der argumentative Modus von Politik in den Vordergrund gerückt wird: „For a deliberative theory, in contrast, it is crucial that citizens (and their representatives) test their interests and reasons in a public forum before they decide. ${ }^{659}$ Damit wird die Rationalität der Ergebnisse von Politik unmittelbar von der „epistemischen Qualität" der Verfahren abhängig, über die sich die öffentliche Debatte organisiert. Dabei wird mit dem Attribut „öffentlich“ hier nicht nur eine Qualifizierung der Art und Weise vorgenommen, wie Bürger deliberieren, sondern es

56 Dryzek 1999, S. 44.

${ }^{57}$ Vgl. Cohen/Sabel 1997, S. 321 f. und Forst 2001, S. 370-374.

${ }^{58}$ Habermas 1998, S. 166.

59 Bohman 1996, S. 5. 
steht zugleich für einen ganz bestimmten Typus von in diesem Zusammenhang akzeptablen Gründen, die sich dadurch auszeichnen, daß nur solche Gründe als Gründe zählen, die alle Beteiligten sich reziprok-allgemein zumuten können und deren Akzeptanz deshalb auch unter moralischen Gesichtspunkten erwartet werden darf. Deshalb setzt „Öffentlichkeit" in diesem Sinne auch kein über geteilte Werte vermitteltes Kollektiv oder eine Gemeinschaft voraus, sondern bezieht sich unmittelbar auf die kooperativen Anstrengungen aller derjenigen, die über gemeinsame Probleme und die Folgen von Handlungen miteinander verbunden sind, so daß man das Modell deliberativer Politik in seiner Programmatik folgendermaßen charakterisieren kann:

"On the deliberative interpretation, then, democracy is a framework of social and institutional conditions that both facilitates free discussion among equal citizens by providing favourable conditions for expression, association, discussion, and ties the authorization to exercise public power - and the exercise itself - to such discussion, by establishing a framework ensuring the responsiveness and accountability of political power to it." ${ }^{60}$

Diesem normativem Hintergrundverständnis eines „epistemischen Prozeduralismus ${ }^{* 61}$ ist also im Kern die Attraktivität geschuldet, die der Idee deliberativer Politik im Rahmen einer institutionellen Übersetzung einer, ,politics of the common good ${ }^{662}$ zukommt. Freilich sind damit mindestens zwei grundlegende Bedenken verbunden, die zum einen die Frage betreffen, ob es angesichts notorischer Dissense überhaupt einen Sinn macht, an der Unterstellung einer konsensgenerierenden argumentativen Praxis festzuhalten (3.1); zum anderen geht es um das dornige Problem, ob wir nicht mit deliberativ erzeugten Ungleichheiten und Exklusionen rechnen müssen, an deren sozialstruktureller Massierung der Gedanke eines rational vermittelten Egalitarismus auflaufen müßte: Dem möchte ich so weit nachgehen, bis unter Rückgriff auf John Deweys epistemische Begründung einer Praxis „reflexiver Kooperation" dem deliberativen Modell eine Fassung gegeben werden kann, die diesen Einwänden standhalten kann (3.2). Dabei geht es darum, in normativer Hinsicht plausibel zu machen, daß das Modell deliberativer Politik in besonderer Weise geeignet ist, die Beweislasten für die These zu übernehmen, $\mathrm{da} ß$ die Idee demokratischer Selbstbestimmung ohne Ermäßigung ihrer normativen Gehalte auf die komplementären Prozesse der Entstaatlichung (Enthierarchisierung) und der Denationalisierung von Politik eingestellt werden kann.

\subsection{Zur Idee des epistemischen Prozeduralismus}

Auch wenn man nicht im Sinne einer starken prozeduralistischen Fassung davon ausgeht, daß die Idee deliberativer Politik eine Vorstellung reiner und vollständiger Verfahrensrationalität im Rawlsschen Verständnis impliziert, die es erlaubte, sie an die Stelle politischer Entscheidungsverfahren (etwa der Mehrheitsregel) zu setzen, sondern ihr lediglich eine rationalisierende Wirkung auf die eigentliche Entscheidung zuschreiben möchte, setzt das immer noch voraus, daß die Beteiligten ihre Interaktion als einen

${ }^{60}$ Cohen/Sabel 1997, S. 320.

61 Estlund 1997.

62 Vgl. Goodin 1996. 
gemeinsamen Lernprozeß organisieren, der unter der Prämisse einer zwar aktuell nicht gefundenen, aber im Prinzip doch erreichbaren richtigen Antwort auf moralische und praktische Probleme steht. Gegen diese Unterstellung, daß Beteiligte sich auf einen Diskurs einlassen, ihre Differenzen zwanglos durch den Bezug auf gute Gründe auflösen und so einen handlungsleitenden Konsens erzielen ${ }^{63}$ sprechen nun zweifellos gewichtige Gründe. Sie liegen zum einen in der „Phänomenologie fortdauernder Kontroversen $^{\text {"64 }}$ und zum anderen in einer Art „Dialektik des Diskurses", wonach die Gefahr, daß es nicht zu einem Konsens kommt, sogar um so größer ist, ,je drastischer die Lerneffekte ausfallen, die [im Rahmen deliberativer Verfahren, R. S.-B.] durch Argumentation zwanglos erzwungen werden", ${ }^{65}$ eine Dialektik, die den Diskurs, wo nicht als soziales, so doch als politisches System sprengen könnte. Weshalb, so lautet deshalb die skeptische Frage, sollte man angesichts solcher Befunde an der im Rahmen des Modells deliberativer Politik in legitimationstheoretischer Hinsicht entscheidenden Prämisse einer richtigen Antwort festhalten? ${ }^{66}$

Dafür sprechen, noch im Vorfeld organisatorischer und institutioneller Fragen, ${ }^{67}$ in normativer Hinsicht insbesondere zwei Gründe. Das erste Argument für eine in einem schwachen Sinne epistemische Auslegung der Legitimationsgrundlagen demokratischer Politik entwickelt Habermas in Beantwortung der Frage, was passieren würde, wenn die Beteiligten ,nicht auch - gewiß in dem fallibilistischen Bewußtsein, sich jederzeit irren zu können - davon ausgehen würden, daß die strittigen und rechtlichen Probleme eine ,richtige' Lösung finden könnten““ ${ }^{68}$ Diese Unterstellung hält Habermas nämlich trotz aller entgegenstehenden empirischen Evidenzen letztlich deshalb für alternativlos, weil wir ohne sie gar nicht verstehen könnten, weshalb Subjekte überhaupt in reziproker Einstellung annehmen können, daß sie ihre Konflikte gewaltlos in dem weiten Sinne bereinigen können, daß sie nicht lediglich auf Mittel des Zwangs, des überlegenen Einflusses, der Täuschung oder der Verführung angewiesen sind. Das eben würde es ihnen verunmöglichen, die Ergebnisse politischer Auseinandersetzungen auf die freiwillige Akzeptanz der Beteiligten zurückzuführen, in der sich ihre wechselseitige Anerkennung als Freie und Gleiche zum Ausdruck bringt; und umgekehrt kann sich das wechselseitige Vertrauen in die überlegene Rationalität gewaltloser Verständigung nur dann entfal-

${ }^{63}$ Vgl. van den Daele/Neidhardt 1996, S. 38.

${ }^{64}$ Habermas 1996b, S. 323.

65 Van den Daele/Neidhardt 1996, S. 40.

${ }^{66}$ Vgl. auch Habermas 1996b, S. 323-328.

${ }^{67} \mathrm{Daß}$ den Fragen des Verfahrensdesigns eine große Bedeutung zukommt, wenn man klären will, unter welchen Bedingungen Argumentation auch zu politisch wirksamen Konsequenzen führt, darauf hat mit Blick auf die empirischen Beispiele, auf die sich van den Daele und Neidhardt stützen, vor allem Saretzki (1996 a und b) insistiert. Es gibt dann keinen Anlaß, die Wirkungen moralischer und argumentativer Orientierungen in der Politik zu unterschätzen. Allerdings setzt eine soziologisch informierte Analyse der Bedingungen voraus, daß man Kontexte und Prozeduren angeben kann, „die es möglich machen, daß moralische Orientierungen, wenn sie generiert werden, auch operativ werden können“ (Giegel 1999, S. 188). Allgemein zu den politisch-institutionellen Perspektiven deliberativer Politik vgl. auch Schmalz-Bruns (1995, S. 167-211).

${ }^{68}$ Habermas 1996b, S. 326. 
ten, wenn alle die Auffassung teilen, daß (gute) Gründe einen epistemischen „Zwang ““ transportieren. ${ }^{69}$

Dieses erste, eher defensive Argument insistiert also darauf, daß der demokratische Rechtsstaat seine Legitimationsgrundlage verliert, wenn wir nicht in der Perspektive der Möglichkeit einer rationalen Überzeugung anderer handeln. Neben diesen legitimationstheoretischen tritt aber noch ein zweiter Grund, der erkennbar wird, wenn man die Funktion in den Blick nimmt, die dem „epistemischen Prozeduralismus“ in gegenmajoritärer Hinsicht zukommen kann. In diesem Zusammenhang macht Estlund zu Recht darauf aufmerksam, daß wir auch im Falle verfahrensmäßig korrekt zustandegekommener und insoweit legitimer politischer Entscheidungen auf verfahrensexterne Legitimationsstandards epistemischer Rationalität gerade dann nicht verzichten können, wenn wir erklären wollen, weshalb wir von Minderheiten nicht erwarten, sich in der Beurteilung politischer Fragen vollständig von prozedural legitimierten Ergebnissen abhängig zu machen. ${ }^{70}$ Der politische Grund dafür liegt in der Unvollständigkeit von Verfahren, die für sich genommen eine auch in substanzieller Hinsicht richtige Entscheidung nicht verbürgen können. Daher nehmen verfahrensexterne Standards eine katalytische Funktion für die Kritik aktueller Verfahren in der Weise an, daß sie die Suche nach Verfahren anregen, denen ein größerer ,epistemischer Wert ${ }^{\text {“71 }}$ in dem Sinne einer Erhöhung der Wahrscheinlichkeit von reziprok-allgemein zu rechtfertigenden Ergebnissen zukommt: Das muß nicht zwangsläufig, wie man befürchten könnte, ${ }^{72}$ auf die Substituierung prozeduraler durch epistemische Legitimität und die Ersetzung der Demokratie durch eine Expertokratie hinauslaufen, sondern es eröffnet uns durch die Vermittlung prozeduraler und epistemischer Dimensionen des demokratischen Prozesses eine Perspektive, in der wir auch dessen institutionelle Logik und Dynamik, die Art des $\mathrm{Zu}$ sammenspiels von politischen Institutionen und demokratischen Öffentlichkeiten sowie das Verhältnis von Mehrheit und Minderheit besser verstehen können. ${ }^{73}$

\subsection{Deliberative Ungleichheiten und die Idee „reflexiver Kooperation“}

Freilich ist damit die weitergehende Befürchtung noch nicht ausgeräumt, daß die den demokratischen Prozess unterminierenden sozialen Ungleichheiten in bezug auf Bildung, Status, Einkommen, Macht und Einfluss durch im engeren Sinne „deliberative Ungleichheiten ${ }^{\text {c } 74}$ nicht noch verstärkt werden könnten. Durch das besondere Gewicht, das in dem Modell deliberativer Demokratie auf einer im öffentlichen Austausch von Gründen vermittelten politischen Teilhabe ruht, sind es neben den durch Machtungleichgewichte asymmetrisch verteilten Zugangschancen zur Öffentlichkeit insbesondere kommunikative Ungleichheiten, die eine effektive Inanspruchnahme formal gleicher Beteiligungsrechte unterlaufen könnten. Diese können insbesondere in der Privilegie-

69 Ebd., S. 323-326.

70 Estlund 1997, S. 183, 184.

${ }^{71}$ Ebd., S. 174.

72 Vgl. Forst 2001, S. 373, Anm. 12.

73 Vgl. dazu Bohman 1996, S. 182-187.

${ }^{74}$ Ebd., S. 110, 112-132. 
rung eines bestimmten Typus diskursiver Rationalität hervortreten, die dann andere (gruppen- oder kulturspezifische) Sprachstile und Ausdrucksverhalten disqualifiziert ${ }^{75}$ und damit ganze Gruppen von einer öffentlich wirksamen Artikulation ihrer Interessen abschneidet. ${ }^{76}$ Eine Antwort auf dieses Problem eines deliberativen Elitismus, das vor allem im Gegenlicht der im Rahmen des Modells deliberativer Politik an die Beteiligten gerichteten Rationalitäts- und Kompetenzerwartungen entsteht, kann in zwei Richtungen gesucht werden: Zum einen kann man bedarfsseitig das Niveaugefälle zwischen theoretisch induzierten und praktisch erwartbaren Motiven, Interessen und Kompetenzen dadurch abschwächen, daß die zugrundeliegenden Rationalitätsstandards nicht im Blick primär auf die Anforderungen einer dem wissenschaftlichen Diskurs nachgebildeten, rein argumentativen Verständigung, sondern auf die Erfahrung sozialer Kooperation in der Lösung gemeinsamer Probleme hin ausgelegt werden (2.); zum anderen wird deliberative Politik auch in dieser etwas schwächeren Fassung nicht ohne geeignete Institutionen und assoziative Arrangements auskommen, die diese diskursiven Orientierungen anregen und gegen Ausbeutung abschirmen (1.). Auf beide Punkte gehe ich kurz in umgekehrter Reihenfolge ein.

(1.) Wenn es unter Bedingungen von Freiheit und Gleichheit normativ gesehen unzulässig ist, das Kriterium (umstrittener) moralischer Überlegenheit der Beteiligten für Zwecke der Rechtfertigung der Legitimität politischer Entscheidungen zu verwenden, dann folgt daraus auch, daß bestehende individuelle Kompetenzunterschiede oder soziale Ungleichheiten sich auch im Licht einer prozedural-epistemischen Auslegung der Legitimationsgrundlagen demokratischer Politik nicht in differentielle Zugangs- und Beteiligungschancen übersetzen dürfen. Da aber gleichwohl deliberative Verfahren unvermeidlich mit höheren Anforderungen an individuelle Motivationen, Interessen und Kompetenzen verbunden sind als rein aggregative Verfahren der Willensbildung- und Entscheidungsfindung, liegt die Beweislast auf der Seite nicht der Individuen, sondern der Verfahren und Institutionen, die vorhandenen Kompetenzen und moralischen Vermögen der Beteiligten in geeigneter Weise anzusprechen. ${ }^{77}$ Ohne darauf hier in aller Ausführlichkeit eingehen zu können, möchte ich kurz vier Merkmale nennen, an denen sich entscheidet, ob Institutionen oder Assoziationen dieser katalytischen Funktion nachkommen können: Als erstes Kriterium kann man jenes der Inklusivität nennen, das jene Verfahren auch in epistemischer Hinsicht auszeichnet, die keine auf askriptiven Merkmalen oder sozialstrukturell induzierten und auf Machtasymmetrien basierende Zugangshürden errichten, sondern für alle individuellen Beteiligungswünsche offen sind; im unmittelbaren Zusammenhang steht damit zweitens, daß horizontale im Unterschied zu vertikalen Formen der Willensbildung besser geeignet sein dürften, der Multiperspektivität und Multivalenz der von Beteiligten im Rahmen von Verständigungsoder kooperativen Problemlösungsprozessen geltend gemachten Gesichtspunkte im Hinblick auf das Verständigungsziel auch gerecht zu werden $;{ }^{78} \mathrm{ob}$ sich aber diskursive oder dialogische Orientierungen in politischen Kontexten durchsetzen können, hängt

\footnotetext{
75 Vgl. Young 1996, S. 122-125.

76 Vgl. Sanders 1997, S. 348-350.

77 Estlund 1997, S. 190.

78 Vgl. Offe 1997, S. 103 f.
} 
drittens auch davon ab, inwieweit es gelingt, die für Verhandlungen typische Amalgamierung von strategischen und diskursiven Orientierungen zu verhindern und das Kompatibilitätsproblem zu lösen, das darin besteht, daß die bloß einseitige Orientierung an Verständigung und an unparteilichen moralischen Gesichtspunkten in der Regel zu einer Schwächung der eigenen Position führt - soll also die Ausbeutung moralischer Orientierungen in aufgrund ihrer strategisch-moralischen Doppelbestimmung unklaren Handlungssituationen vermieden werden, müssen sie gegen das Eindringen strategischer Orientierungen von vornherein abgeschirmt werden; ${ }^{79}$ schließlich qualifizieren sich Verfahren vor allem dann für die o. g. katalytische Funktion, wenn die Möglichkeit der Erhebung „reziproker Einwände ${ }^{\text {"80 }}$ institutionalisiert ist - gerade wenn in konkreten Willensbildungs- und Entscheidungskontexten wirklich allgemeine Partizipation nicht zu gewährleisten und eine wirklich allgemeine Akzeptanz der Ergebnisse nicht zu erreichen sein dürfte, ist es um so wichtiger, auch nachträglich Einwände zuzulassen, sofern sie geltend machen können, daß reziprok-allgemein nicht zurückweisbare Ansprüche im Verfahren ignoriert worden sind.

(2.) Ganz in diesem Sinne hat etwa Estlund denn auch vorgeschlagen, nur wenige und zudem basale soziale und strukturelle Kriterien in Anschlag zu bringen, die den ,epistemischen Wert" von auf diese Weise institutionell eingehegten Verfahren hinreichend verbürgen können sollen. Dazu rechnet er u. a. die Normen, daß

„1. [e]very adult in society is permitted to participate. 2. Participants sincerely address questions of justice, not of interest group advantage, and it is common knowledge that this is so. [...] 4 . Participants evaluate arguments fairly, irrespective of the identity of the person, or the size of the group offering the argument. 5. Each participant's views are easily available to others $[\ldots]$ 6. Participants represent a personal, educational, and cultural variety of life experiences. 7. Participants' needs for health and safety are sufficiently well met that it is possible for them to devote some time and energy to public political deliberation, and in general all are literate. ${ }^{.481}$

Durch diese Art der institutionellen Vorsorge dürften nun zwar Bedenken hinsichtlich der exkludierenden Effekte deliberativer Verfahren einigermaßen auszuräumen sein, aber doch um den Preis, daß dann umgekehrt eine Spannung zu den Rationalitätsunterstellungen einer nicht nur über Gründe schlechthin, sondern über ausreichend gute Gründe vermittelten Praxis der demokratischen Selbstbestimmung auftreten. Das legt es nahe, eine neue Balance zwischen diesen beiden Polen zu finden, indem man mit Dewey die einschlägigen Rationalitätserwartungen nicht allein an der intersubjektiven Rede, sondern an der Erfahrung gemeinschaftlicher Kooperation entbindet, ${ }^{82}$ was beides gewährleisten soll: die über den gemeinschaftlichen Problemlösungsbezug sich einstellende pragmatische Qualifizierung ,ausreichend guter Gründe ${ }^{483}$ und damit unmittelbar

79 Giegel 1999, S. 189 f.; vgl. auch Anm. 3.

${ }^{80}$ Forst 2001, S. 370.

${ }^{81}$ Estlund 1997, S. $190 \mathrm{f}$.

82 Vgl. Honneth 1999, S. 61.

${ }^{83}$ Das ist der Vorteil, den auch Bohman (1996, S. 27) heraushebt, wenn er notiert: „However, one of the main advantages of seeing deliberation primarily as a cooperative activity is that the standard of publicity need not depend on strong idealizations that do not refer to any actual deliberation ${ }^{c \text {, }}$, so 
verbunden den hinreichend plausiblen Nachweis von Interessen und Motiven, sich an der öffentlichen Meinungs- und Willensbildung überhaupt zu beteiligen. ${ }^{84}$

Dieses Ziel erreicht Dewey einerseits durch eine epistemische Rechtfertigung der Demokratie, in der diese zur Voraussetzung der vollständigen Anwendung des geistigen Vermögens der Bürger auf die intelligente Lösung gesellschaftlicher Probleme wird, andererseits mit einem anti-paternalistischen Zug verbindet: Er besteht in diesem Sinne darauf, daß die Bestrebungen zur Förderung des gemeinsamen Guten nur dann gut sein können, wenn sie nicht auf Kosten der aktiven Entwicklung der Nutzenempfänger gehen, und nur dann gemeinsam, wenn diese auch einen effektiven Anteil an der Herbeiführung des Resultats haben. ${ }^{85}$ Genau dieser übergreifenden Aufgabenbestimmung verdankt sich denn auch sein ,konsequentialistischer" Öffentlichkeitsbegriff, ${ }^{86}$ in dem Dewey versucht, sein methodisches Ideal einer ,social inquiry“ in ein mit fundamentalen Egalitätsnormen verträgliches Modell epistemischer Politik so zu übersetzen, daß die in öffentlicher Deliberation vermittelte demokratische Praxis der kollektiven Selbstbestimmung in funktionaler Hinsicht zugleich selbstkonstitutiv und in ethischer Hinsicht selbstransformativ gedacht werden kann. ${ }^{87}$ „Öffentlichkeit" konstituiert sich für Dewey zunächst über

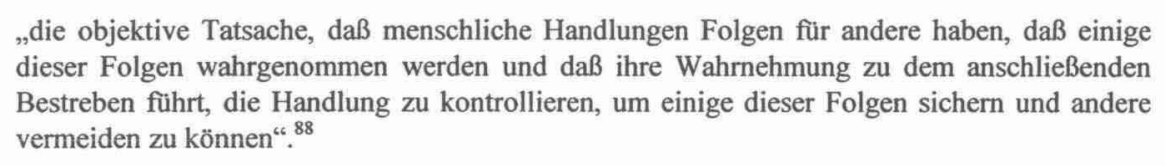

Dieser für das pragmatistische Denken charakteristische konsequentialistische Impuls ${ }^{89}$ ist dafür maßgeblich, daß Dewey den Blick zurücklenkt auf jene „lokalen“ (oder auch: sektoralen) Kontexte der Problemgenese und -lösung, die ihm zudem im Sinne einer partizipatorischen Demokratie noch am ehesten Gewähr dafür zu bieten scheinen, die gleichberechtigte Teilhabe aller an den öffentlichen Angelegenheiten zu ermöglichen - aus diesem Grund nimmt „Öffentlichkeit" für ihn die Gestalt von vielfach funktional, sektoral oder lokal differenzierten Öffentlichkeiten und Teilöffentlichkeiten an, die aber horizontal und vertikal, über die Ebenen des Sozialen und des Politischen sich erstreckend, miteinander verknüpft sind. Freilich ist durch ein gemeinsames Kontrollinteresse allein am Ende noch nicht sicherzustellen, daß alle Beteiligten sich etwa darüber einigen könnten, wie richtig

daß ein kooperationsbasierter Ansatz verspricht, ,[to] provide both an epistemic and a moral basis for democratic participation in complex societies".

${ }^{84}$ Honneth 1999, S. 64 f.

${ }^{85}$ Putnam 1997, S. 232.

86 Vgl. Kettner 1998, S. 58-62.

${ }^{87}$ Diese Programmatik liegt auch der Entwicklung einer post-positivistischen Policy-Analyse zu Grunde, die als „policy science of democracy“ (Lasswell) von der Policy-Forschung verlangt, ,ein anderes Wissen an[zu]bieten als bisher, [...] ein anderes Verhältnis zu ihren Forschungsobjekten und -adressaten ein[zu]nehmen und für eine offenere Vermittlung ihrer Ergebnisse in den Politikprozeß ein[zu]treten“" (Saretzki 1999a, S. 301f).

${ }^{88}$ Dewey 1996, S. 26 f.

89 Vgl. auch Knight/Johnson 1999, S. 567. 
$\mathrm{zu}$ kontrollieren sei ${ }^{90}$ und wie sich die unterschiedlichen Perspektiven von Beteiligten und Betroffenen verschränken. Deshalb wird für Dewey die Frage der Transformation der „great society“ in eine "great community" zu dem Bezugsproblem, dem sich die Entfaltung seines Modells einer in epistemischen Orientierungen vermittelten reflexiven Kooperation verdankt: In der Vorstellung von demokratischer Selbstbestimmung nach dem Modell einer "social inquiry" entfaltet Dewey seine spezifische Idee kommunikativer Vergemeinschaftung (2.1), die zugleich eine Brücke bildet zwischen dem menschen- und staatsbürgerrechtlich verankerten Egalitätsgebot in der Kantischen Tradition einerseits und der epistemischen Begründung der Demokratie in der Tradition von J. S. Mill andererseits; ${ }^{91}$ von hier aus ergeben sich relativ zwanglos sein mit der Idee der Öffentlichkeit intern verbundener Begriff von Gemeinschaft (2.2.) wie seine in der Idee reflexiver Kooperation vermittelte Vorstellung einer gemeinwohlbezogenen Politik (2.3.) - diese Schritte möchte ich im Folgenden jeweils knapp erläutern.

(2.1.) Deweys Versuch zu zeigen, wie sich im Horizont kooperativer Problemlösung die Momente von Pluralität, Gleichhcit und Freihcit zu einer "epistemischen“ Legitimationsgrundlage und zu einer andersartigen Organisationsform der Praxis demokratischer Selbstbestimmung verbinden, ist kategorial im Begriff, ,vollkommener Erfahrung “ zentriert, den er zunächst im Zusammenhang seiner ästhetischen Theorie entwickelt ${ }^{92}$ und dann im Kommunikationskapitel seines naturphilosophischen Werks über „Erfahrung und Natur ${ }^{\star 93}$ so aufgreift, daß er nunmehr auch in der alltäglichen Kommunikation ein Ideal entdecken kann, an dem sich genau die normativen Gehalte entfalten lassen, die er dann dem (oft einseitig instrumentell missverstandenen) Prozeß einer demokratischen "social inquiry“ einschreibt. ${ }^{94}$ Wesentlich ist dabei, daß sich in der Kommunikation stets instrumentelle und finale ("consummatory") Aspekte zu einer Form des Austauschs verbinden, "der $\mathrm{zu}$ der Befriedigung eines Bedürfnisses verhilft" 95 weil „Kommunikation [...] auf einzigartige Weise sowohl Mittel wie Ziel [ist]. Sie ist Mittel“, wie er emphatisch festhält, „Kooperation, Herrschaft und Ordnung zu bewerkstelligen. Gemeinsame Erfahrung ist das größte unter allen menschlichen Gütern" ${ }^{\text {" } 96}$ - und in dieser Gestalt verknüpft er den Begriff vollkommener Erfahrung dann mit seiner spezifischen Form der Rechtfertigung der Demokratie, die er eben nicht

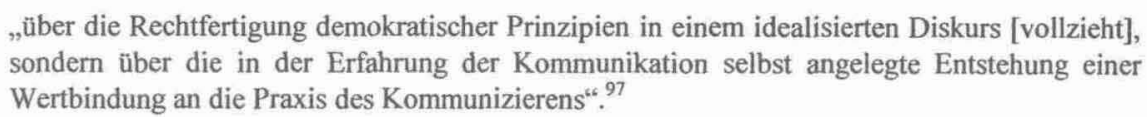

Erst nachdem Dewey seine Vorstellung demokratischer Praxis in dem normativen Begriff von Kommunikation verankert hat, kann er dann den für Demokratie als soziale

\footnotetext{
${ }^{90}$ Vgl. Kettner 1998, S. 60 f.

91 Vgl. MacGilvray 1999, S. 553.

92 Vgl. dazu auch die Ausführungen in Schmalz-Bruns 1998b, S. 431-438.

93 Dewey 1995, S. 167-204.

94 Vgl. Joas 1997, S. 184-187.

95 Dewey 1995, S. 182.

96 Ebd., S. 201.

97 Joas 1997, S. 187, Hervorh. R. S.-B.
} 
Lebensform konstitutiven Zusammenhang zwischen Freiheit, Kommunikation, kooperativer Wissenserzeugung und gemeinschaftlicher Problemlösung auf dreierlei Weise herstellen: Zunächst macht er geltend, daß sich die Erfüllung des individuellen Lebens nur unter Bedingungen einstellen kann, die durch die gleichberechtigte, freie und aktive Teilhabe am gesellschaftlichen Leben gekennzeichnet sind; während die liberale Demokratie mit der durchgesetzten Trennung der Sphären von Alltag, Arbeit und Politik diese Anforderungen unterläuft, schneidet die repräsentative Demokratie die einzelnen auch von den intrinsisch befriedigenden Erfahrungen gemeinsamen, auf die Lösung kollektiver Probleme bezogenen Handelns ab; und sie verhindert in ihrer Fixierung auf die institutionellen Strukturen staatlicher Politik die Herausbildung einer Öffentlichkeit, in der gegensätzliche Meinungen so miteinander in Bezug gesetzt werden können, daß Vielheit und Pluralität auch in einem kognitiven Sinn fruchtbar gemacht werden können. ${ }^{98}$ In dieser Zuspitzung jedenfalls wird sichtbar, wie Dewey, vermittelt über die Brückenkonzepte von „Kommunikation" und „vollendeter Erfahrung", eine interne Verbindung der epistemisch-diskursiven Aspekte des Prozesses kollektiver Problemlösung (und damit auch der dem wissenschaftlichen Forschungsprozeß entnommenen methodischen Orientierung) einerseits und der Idee der Demokratie andererseits herzustellen versucht, die geeignet ist, seine Vorstellungen gegenüber expertokratischen Verdächtigungen abzuschirmen: Es geht ihm, kurz gesagt, nicht nur darum, ,[to solve] the enlightenment problem of making democracy more like science, but also the political problem of making science more like democracy“. ${ }^{99}$ In diesem Sinne stellt sich für Dewey denn auch ein doppelter Bezug zwischen der Idee eines methodisch angeleiteten Prozesses der Problemlösung und der aus Beteiligten und Betroffenen sich bildenden öffentlichen (Kommunikations-) Gemeinschaft her. Die „social inquiry“ bleibt ebenso in dem Boden eines gemeinschaftlich erzeugten Wissensbestandes verankert, wie umgekehrt die Anstrengungen zur Problemlösung nicht nur aus dem gemeinschaftlichen Leben herauswachsen, sondern auf dieses verändernd zurückwirken: Zum einen in interner Perspektive in dem Sinn, daß die Erfahrung von Kommunikation selber ethische Einsichten erschließt, die die Vorstellung einer gemeinschaftlichen Lebensform durchdringen und prägen; zum anderen in externer Perspektive dadurch, dass im Zusammenhang einer kumulativen Wissensgenese und -anwendung auch die Horizonte der Zugehörigkeit zur Gemeinschaft permanent verschoben werden. ${ }^{100}$ Insofern läßt sich Deweys Vorstellung einer epistemischen, experimentellen Demokratie in sieben Aspekten zusammenfassen: ${ }^{101}$ Was in pragmatistischer Perspektive an der Praxis einer methodisch kontrollierten Form kollektiver Problemlösung interessiert, ist nicht nur die Überzeugung, daß „,inquiry [is] the best way to settle doubt and justify belief in the face of unexpected contingencies" ${ }^{102}$ sondern darüber hinaus die gemeinschaftsbildende und wertbindende Kraft der Erfahrung öffentlicher Kommunikation; die „social inquiry“ ist deshalb wissenschaftlich nur in dem Sin-

\footnotetext{
98 Diese Argumentationslinie hat Dewey 1996 in Die Öffentlichkeit und ihre Probleme systematisch entfaltet; zum Argumentationsplan dieser Schrift vgl. Schmalz-Bruns 1995, S. 213-220.

99 Bohman 1999, S. 591.

${ }^{100}$ Vgl. Smiley 1999, S. 632.

${ }^{101}$ Hier stütze ich mich auf Knight/Johnson 1999, S. 574 f.

${ }^{102}$ Ebd., S. 574.
} 
ne der Anwendung geeigneter Verfahren und Methoden der kollektiven Wissenserzeugung und -vermittlung, und sie ist nicht auf die Verfolgung eines naturwissenschaftlichen Methodenideals und mithin auf die expertokratische Enteignung des Prozesses kollektiver Selbstbestimmung hin ausgelegt; das hat zur Folge, daß vor dem Hintergrund eines für den pragmatistischen Ansatz charakteristischen Fallibilismus auch die Ergebnisse der social inquiry als im Licht sozialer Erfahrungen und politischer Debatten grundsätzlich revisionsoffen verstanden werden müssen; aus diesem Grund bleiben, im Sinne einer bis zu einem gewissen Grade unvermeidlichen Arbeitsteilung zwischen Experten die instrumentellen Aspekte des Forschungsprozesses stets eingebunden in öffentliche Debatten über kollektive Ziele und Zwecke, so daß,,the results of inquiry must enter into social and political deliberation and debate rather than be applied to problems ${ }^{6{ }^{6103}}-\mathrm{m}$. a. W. bleibt die allgemeine politische Öffentlichkeit der Ort, an dem sich Forschung und soziale Zwecksetzung, instrumentelle und finale Aspekte der Deliberation zu Zwecken der Problemlösung kommunikativ durchdringen und damit jene gemeinschaftsbildenden Potentiale freisetzen, die Dewey der Erfahrung der Kommunikation zumißt. $^{104}$

(2.2.) Dem entsprechen nun spiegelbildlich Deweys Vorstellung der Organisationsform einer neuen Öffentlichkeit wie das daran sich ausrichtende Verständnis von politischer Vergemeinschaftung - einer neuen Öffentlichkeit also, die in einem Netzwerk sich wechselseitig überlappender, dezentraler Problemlösungsgemeinschaften jene fokalen Interaktionszusammenhänge auf neuer Stufe wiederherstellen soll, die konstitutiv für die Entfaltung einer zugleich egalitären und epistemisch fundierten Praxis der Selbstbestimmung sind. Vor diesem Hintergrund wird nun auch deutlich, daß Dewey seinem Begriff von Gemeinschaft kein im Sinne vorgängiger und dem politischen Prozeß exogener Wertbindungen ethisches Verständnis zu Grunde legt; sondern er zentriert sein Gemeinschaftsverständnis ganz entschieden funktional in einem über Folgen vernetzten, interdependenten Handlungszusammenhang, dem in erster Linie ein moralisch gestimmtes Interesse an den Wirkungen eigener Handlungen auf andere Beteiligte und Betroffene wie an der wechselseitigen Anerkennung der Autonomie aller in diesen Handlungszusammenhang hineingezogenen Individuen oder Gruppen entspricht. ${ }^{105}$ Diese Vorstellung einer ,moral community“ als Bezugspunkt des voll entfalteten, pragmatistischen Ideals demokratischer Selbstbestimmung hat etwa Smiley deutlich herausgearbeitet, indem sie feststellt:

\footnotetext{
${ }^{103}$ Ebd., S. 575.

${ }^{104}$ Deshalb insistiert Morris 1999, S. 619-623, auch zu Recht darauf, dass sich die spezifischen Gehalte der Vorstellung Deweys nur mit Bezug auf die Unterscheidung von „inquiry“ einerseits und "discovery" andererseits herausstellen lassen: Erst die Stilisierung des Prozesses der Problemlösung als eines öffentlichen Entdeckungszusammenhangs macht darauf aufmerksam, dass es Dewey im Rahmen der gemeinschaftlichen Transformation der "great society" in erster Linie auf die Beschreibung von „more complex ends“ ankommt. In diesem Sinne ist sein Punkt „only incidentally an epistemological one. It is rather more radical - to commend science, art, and democratic community as productive not only of factual knowledge, but of meaning" (Morris 1999, S. 621).

${ }^{105}$ Vgl. Knight/Johnson 1999, S. 573.
} 
„Dewey himself appears to have accomplished three things by including the poor and members of other nations in our community of interests. First of all, he appears to have rendered their suffering, our business'. Second, he appears to have shown that our economic system has more consequences than many of us now realize. Third, he appears to have incorporated those suffering into our ,public' (i. e. into that group whose interests we take into consideration when tracing the consequences of our actions). All three accomplishments necessitated that Dewey both develop an expanded notion of community and convince us to accept that conception ourselves. " 106

Dieses Verständnis folgt gewiß dem unvermeidlich universalistischen Zug, der in die Idee demokratischer Selbstbestimmung, basierend auf dem Prinzip des wechselseitigallgemeinen Rechts auf Rechtfertigung, eingelassen ist; und insofern ist es inklusiv, als die Charakterisierung einer idealen Öffentlichkeit durch die bewußte Hinwendung auf die Folgen von Handlungen uns im Licht dieses Konsequentialismus dazu nötigt, permanent im Medium des öffentlichen Diskurses die Grenzen unserer Gemeinschaft neu zu ziehen (und zu erweitern). Freilich zieht dieser Modus der Inklusion unmittelbar zwei Probleme nach sich, auf die eine Antwort gefunden werden muß: Zum einen das Problem gleichsam einer inneren Arbeitsteilung zwischen Experten und Laien innerhalb der Forschungsgemeinschaft, die sich nicht zu einer Marginalisierung der Laien auswachsen darf, wenn der demokratische Anspruch nicht beschädigt werden soll; und zum zweiten die Frage, ob sich der konsequentialistische Begriff von Öffentlichkeit und das Gemeinschaftskonzept tatsächlich so vollständig fusionieren lassen, wie Dewey sich das vorstellt - denn wenn die Reichweite und Bedeutung von Konsequenzen, auf die sich das gemeinschaftliche Handeln richten soll, immer auch von Interpretationen abhängig ist, dann könnten wir es mit einem Henne-Ei-Problem derart zu tun haben, daß sich die Frage aufdrängt, was zuerst kommt: „the community or its acknowledgement of consequences"? ${ }^{107}$

(2.3.) Auf beide Probleme, und insbesondere auch auf das allgemeine Konstitutionsproblem der demokratischen Erzeugung einer demokratischen Forschungs-) Gemeinschaft kann man nur reagieren, wenn man Deweys Idee einer Kooperationsgemeinschaft um die Idee reflexiver Kooperation erweitert, die schließlich auch den Schlüssel für die Beantwortung der Frage bereithält, wie die neuen Formen der politischen Zwangsvergemeinschaftung jenseits des Nationalstaates im Zeichen eines gemeinwohlorientierten Handelns aller Beteiligten und Betroffenen auch normativ integriert werden könnten. Diese Idee läßt sich wiederum in drei (hier nur anzudeutenden) Schritten entfalten. Zunächst basiert sie auf jener schon herausgestellten Vorstellung einer kooperationsmoralischen Stiftung von Gemeinschaft ein, die Honneth folgendermaßen charakterisiert: Wenn wir die demokratischen Verfahren als Funktion der kooperativen Problemlösung verstehen wollen, dann setzt die „Wiederbelebung von demokratischen Öffentlichkeiten unter Bedingungen komplexer Industriegesellschaften mithin eine Reintegration der Gesellschaft voraus, die nur in der Entwicklung eines gemeinsamen Bewußtseins der subpolitischen Assoziation aller Bürger bestehen kann ${ }^{\text {“ } 08}$ - und diesen Mechanismus

\footnotetext{
${ }^{106}$ Smiley 1990, S. 376.

${ }^{107}$ Smiley 1999, S. 636 und S. 642.

${ }^{108}$ Honneth 1999, S. 58.
} 
der subpolitischen Erzeugung einer demokratischen Sittlichkeit verankert Dewey, wie gezeigt, in der ins Politische geweiteten Idee sozialer Arbeitsteilung, die ,,jedem einzelnen Gesellschaftsmitglied ein Bewußtsein davon geben kann, kooperativ mit allen anderen zur Verwirklichung gemeinsamer Ziele beizutragen“. ${ }^{109}$ Daneben tritt dann - zweitens - die epistemische Deutung dieser Kooperationsbeziehungen, mit der Dewey die Brücke legt, über die alle Einzelhandlungen in der Perspektive der Beförderung des gemeinsamen Wohls aller verbunden werden sollen, denn das ,gemeinsame Wohl kann nur durch Mittel vorangebracht werden, die auf seiten derjenigen, die Nutzen daraus ziehen oder ,gebessert" werden sollen, positives Interesse und aktive Energie entstehen las$\operatorname{sen}^{\text {"11 }}$ - und gut ist, was die allseitige Entwicklung und Entfaltung der Potentiale aller Beteiligten im Sinne der Ermöglichung konsummativer Erfahrung verbessert. Und an diesen Schritt schließt sich drittens dann die Antwort auf die Frage an, wie die demokratische Autorität unter Bedingungen einer epistemisch durchdrungenen Praxis der kooperativen Problemlösung gewahrt werden kann. Erst mit der Antwort auf diese Frage wird Deweys Kooperationsbegriff nun auch reflexiv, insofern er die Lösung dieses Dilemmas auf die Herstellung deliberativer und Institutionen und Foren projiziert, in denen Fragen der Fairneß und Gerechtigkeit der Kooperationsbeziehungen selber zum Thema werden, indem ,those affected by a decision are able to make judgments about the credibility of experts and to influence the terms of their on-going cooperation with them". ${ }^{111}$

Mit Hilfe dieser in Deweys Öffentlichkeitsbegriff verankerten Vorstellung reflexiver Kooperation kann man im Ansatz plausibel machen, wie eine in einer genuin demokratischen Form von Sittlichkeit verankerte Gemeinwohlorientierung politischen Handelns auch in den neuen, funktional gestifteten Kontexten transnationaler Politik operativ und strukturell befördert werden kann. Dieses so entstehende Bild einer „experimentellen“ Wissensgesellschaft, ${ }^{112}$ mit dem Dewey seiner Vorstellung von deliberativer Politik Konturen zu geben versucht, enthält dann schon die wesentlichen Merkmale, die es schließlich auch für die Konzeptualisierung demokratischer Politik in transnationaler Perspektive als besonders geeignet erscheinen lassen: Es eröffnet den Blick auf Formen politischer Vergemeinschaftung, die nicht mehr notwendigerweise im Horizont geteilter Wertüberzeugungen sich vollzieht, sondern die aus dem Zusammenwirken arbeitsteilig aufeinander bezogener, funktional und sektoral spezifizierter Öffentlichkeiten resultiert und insoweit die nationalstaatlichen Schicksalsgemeinschaften (Held) in ihrer für demokratische Politik konstitutiven Rolle ablöst; ${ }^{113}$ darüber hinaus ist diese neue Organisationsstruktur politischer Öffentlichkeiten auch darauf hin ausgelegt, im Sinne eines

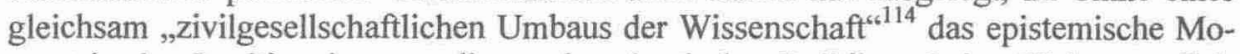
ment in den Legitimationsgrundlagen demokratischer Politik auf eine Weise zur Geltung $\mathrm{zu}$ bringen, die mit Egalitätsnormen kompatibel ist; deshalb bilden schließlich sozial gerechte, kooperationsgemäße Formen der Arbeitsteilung einen internen Bestand-

\footnotetext{
${ }^{109}$ Ebd., S. 59.

${ }^{110}$ Putnam 1997, S. 232.

${ }^{111}$ Bohman 1999, S. 592 und S. 599.

${ }^{112}$ Dewey 1996, S. 169.

${ }^{113} \mathrm{Vgl}$. auch Abromeit/Schmidt 1998.

${ }^{114}$ Kettner 1998, S. 63.
} 
teil seines Demokratieideals, so daß die Forderung sozialer Gleichheit als gleichursprünglich mit der Idee demokratischer Willensbildung verstanden werden muß. ${ }^{115}$

\section{Gemeinwohl und transnationale Politik - strukturelle Implikationen}

Abschließend möchte ich einige Kriterien benennen, denen solche Strukturbildungen auf transnationaler Ebene genügen können müßten, die Gemeinwohl und Gemeinsinn auch unter Bedingungen einer entgrenzten Politik operativ werden lassen. Die in den bisherigen Überlegungen schrittweise entwickelten Anforderungen an einem dem entsprechenden, transnationalen politischen Ordnungszusammenhang lassen sich zusammenfassend zu drei Gruppen sortieren: Berührt sind zum einen Anforderungen, die sich aus einem funktionalen im Unterschied zu einem territorialen Organisationsprinzip transnationaler Politik ergeben und damit Fragen betreffen, die sich auf die Bestimmung von Bezugsgruppen einer politikbereichsspezifischen Willensbildung und Entscheidungsfindung beziehen; daraus ergeben sich zum zweiten spezifisch prozedurale Anforderungen, denen die interne Willensbildung in den unterschiedlichen deliberativen Arenen genügen können muß, um die Gewährleistung der deliberativen und demokratischen Qualität der Verfahren mit sozialisierenden und zivilisierenden Effekten zu verbinden; zum dritten müssen Institutionen vorgesehen werden, die das Management der komplexen Interdependenz der politischen Prozesse in den unterschiedlichen Arenen und das damit verbundene Problem der Kompetenzallokation in den Horizont einer kollektiv-allgemeinen Willensbildung zurückzustellen vermögen. Daraus ergibt sich eine Liste von sehr abstrakten Kriterien:

(1.) Wie Abromeit/Schmidt ${ }^{116}$ zu Recht hervorgehoben haben, sind die Referenzgruppen einer transnationalen Demokratie im Unterschied zu den nationalstaatlichen Demoi durch sechs Merkmale gekennzeichnet: erstens durch Funktionalität/Sektoralität (im Gegensatz zur Territorialität), zweitens durch Latenz, drittens durch Temporalität (im Gegensatz zu Permanenz), viertens durch Subjektivität (statt Objektivität), fünftens durch Prozessualität (statt Substantialität) und sechstens durch Anonymität. Unter diesen Bedingungen kann sich die Referenzgruppenbildung nur nach dem Muster der freiwilligen und ungehinderten Assoziierung nach dem Kongruenz- und Betroffenheitsprinzip vollziehen: Dem entsprechend müßte man die Zugangsbedingungen wie den Status der Willensbildung sichernde und die Interaktion mit anderen sektoralen Demoi regulierende, gleichsam transnational-sektorale Staatsbürgerschaftsrechte konzeptualisieren, wie sie etwa in den Diskussionen um eine „technologische Staatsbürgerschaft“ schon vorgebildet sind ${ }^{117}$ und dies auch mit dem Ziel, eine vollständige „Balkanisierung“ transnationaler Politik zu verhindern.

${ }^{115} \mathrm{Vgl}$. Honneth 1999, S. 63 f.

${ }^{116}$ Abromeit/Schmidt 1998, S. 300.

${ }^{117}$ Vgl. dazu den sehr instruktiven Aufsatz von Saretzki 1999b, der zugleich auf die damit verbundenen vielfältigen Probleme aufmerksam macht. 
(2.) Nach innen steht die Entwicklung eines angemessenen Verfahrensdesigns unter dem Imperativ, nicht nur die epistemische Qualität der Willensbildung unter Vermeidung deliberativer Ungleichheiten zu sichern, sondern zugleich nach Verfahren zu suchen, die weniger von traditionalen Gemeinschaftspotentialen zehren und dafür ein höheres ,gemeinschaftsstiftendes" Potential besitzen. ${ }^{118}$ D. h. sie müssen zugleich eine qualifizierende, eine zivilisierende und eine vertrauensstiftende Wirkung aufweisen: ${ }^{119}$ Qualifizierend in dem Sinne, daß sie die Aktualisierung moralischer Orientierungen von Beteiligten prämieren und gegen strategische Ausbeutung abschirmen, Anreize für die Ausbildung angemessener Kompetenzen vermitteln und so dazu beitragen, „[to] effectively transform the privilege of civic-republican elites into a mental property shared by all citizens"; ${ }^{120}$ zivilisierend im Sinne des Konzepts relationaler Autonomie, die den Beteiligten die von Gutmann und Thompson ${ }^{121}$ herausgehobenen Reziprozitätstugenden der „civic integrity“ und der „civic magnanimity “ ansinnen und diese effektiv vermitteln; und schließlich vertrauensstiftend in dem Sinne institutionell vermittelten Vertrauens unter Fremden, das sich wohl nur unter der Voraussetzung einstellen kann,

,that we share a significant institutional space with a sufficiently strong meaning so as to make the overwhelming majority of ,strangers' among my fellow citizens worthy of being trusted because I anticipate them to be appreciative of that meaning ${ }^{\text {“122 }}$ -

wobei diese Leistung nicht zuletzt von der „moralischen Plausibilität" der Institutionen selber und damit davon abhängt, inwieweit sie die Betroffenen auf die Tugenden des „truth-telling“, der „fairness“, des ,promise-keeping “ und der Solidarität verpflichten. ${ }^{123}$

(3.) Unter Gesichtspunkten von Komplexität treten schließlich die wechselseitig miteinander verknüpften allokativen Fragen nach dem „wer“ (ist legitimer Träger der politischen Willensbildungs- und Entscheidungsprozesse), dem „was" (also den geeigneten Gegenständen politischer Willensbildung) und dem "wie“ (oder den spezifisch geeigneten Verfahren) in den Vordergrund. In diesem Zusammenhang müßte sich die Komplexitätstauglichkeit der Strukturen einer transnationalen Politik insbesondere daran erweisen, inwieweit sie für Formen subsidiärer Differenzierung offen sind; ob sie die Formvarianzen des demokratischen Prozesses, die sich auf einem Kontinuum zwischen den Polen einer direkt-deliberativen Demokratie einerseits und einer rein majoritären Demokratie andererseits ansiedeln lassen, in sich aufnehmen können; und ob sie in der Lage sind, die Spannungen zu verarbeiten, die sich aus dem erforderlichen Neben- und Miteinander dieser funktional irreduziblen Formenvielfalt ergeben.

\footnotetext{
${ }^{118}$ Zürn 1998a, S. 254.

${ }^{119} \mathrm{Vgl}$. auch March/Olsen 1995, S. 45 f.

${ }^{120}$ Offe 1997 , S. 100

${ }^{121}$ Gutmann/Thompson 1996, S. 81-85.

${ }^{122}$ Offe 1999, S.71

${ }^{123}$ Ebd., S. 73-76.
} 


\section{Literatur}

Abromeit, H./Schmidt, Th. (1998), Grenzprobleme der Demokratie: konzeptionelle Überlegungen, in: B. Kohler-Koch (Hg., 1998), S. 293-320.

Beisheim, M./Dreher, S./Walter, G./Zangl, B./Zürn, M. (1999), Im Zeitalter der Globalisierung? Thesen und Daten zur gesellschaftlichen Denationalisierung, Baden-Baden.

Bohman, J. (1996), Public Deliberation. Pluralism, Complexity, and Democracy. Cambridge, MA.

Bohman, J. (1999), Democracy as Inquiry, Inquiry as Democratic: Pragmatism, Social Science, and the Cognitive Division of Labour, in: American Journal of Political Science 43/2, S. 590-607.

Bohman, J./Rehg, W. (Hg., 1997), Deliberative Democracy. Essays on Reason and Politics, Cambridge, MA.

Brunkhorst, H. (Hg., 1998), Demokratischer Experimentalismus. Politik in der komplexen Gesellschaft, Frankfurt/M.

Brunkhorst, H. (1999), Heterarchie und Demokratie, in: Brunkhorst/Niesen (Hg., 1999), S. 373-385.

Brunkhorst, H./Niesen, P. (Hg., 1999) Das Recht der Republik. Frankfurt/.M.

Chwaszca, Ch./Kersting, W. (Ḧg., 1998), Politische Philosophie der internationalen Beziehungen, Frankfurt/M.

Cohen, J. (1997), Procedure and Substance in Deliberative Democracy, in: J. Bohman/W. Rehg (Hg.) 1997, S. 407-437.

Cohen, J. (1998), Democracy and Liberty, in: J. Elster (Hg.) 1998, S. 185-231.

Cohen, J./Rogers, J.(1998), Can Egalitarianism Survive Internationalization? in: W. Streeck (Hg.) 1998, S. 175-193.

Cohen, J./Sabel, Ch. (1997), Directly-Deliberative Polyarchy, in: European Law Journal 3/4, S. 313-342.

Dewey, J. (1934), A Common Faith. New Haven, CT.

Dewey, J. (1995), Erfahrung und Natur. Frankfurt/M.

Dewey, J. (1996), Die Öffentlichkeit und ihre Probleme. Bodenheim.

Dorf, M. C./Sabel, Ch. F. (1998), A Constitution of Democratic Experimentalism, in: Columbia Law Review 98/2, S. 267-473.

Dryzek, J. S. (1999), Transnational Democracy, in: The Journal of Political Philosophy 7/1, S. 30-51.

Eder, K./Hellmann, K.-U./Trenz, H.-J. (1998), Regieren in Europa jenseits öffentlicher Legitimation? Eine Untersuchung zur Rolle von politischer Öffentlichkeit in Europa, in: B. Kohler-Koch (Hg.) 1998, S. 321-344.

Elster, J. (Hg., 1998), Deliberative Democracy, Cambridge/New York.

Elster, J. (1998a), Introduction, in: J. Elster (Hg.) 1998, S. 1-18.

Estlund, D. (1997), Beyond Fairness and Deliberation: The Epistemic Dimension of Democratic Authority, in: J. Bohman/W. Rehg (Hg.) 1997, S. 173-204.

Farr, J. (1999), John Dewey and American Political Science, in: American Journal of Political Science 43/2, S. 520-541.

Forst, R. (2001), The Rule of Reasons. Three Models of Deliberative Democracy, in: Ratio Juris 14/4, S. 345-378.

Frank, M. (1998), Kritik und Metakritik des liberalen Nationalismus. Frankfurt/M.

Frankenberg, G. (1995), Republik und Sozialstaat. Stichworte zum Zusammenhang von öffentlicher Freiheit und ziviler Solidarität, in: Kritische Vierteljahresschrift für Gesetzgebung und Rechtswissenschaft 78/1, S. 25-41. 
Frankenberg, G. (1997), Die Verfassung der Republik. Autorităt und Solidarität in der Zivilgesellschaft, Baden-Baden.

Giegel, H.-J. (1999), Moralische Orientierungen im politischen Prozeß - ein Ankerplatz für die normative Analyse der Demokratie?, in: M. Th. Greven/R. Schmalz-Bruns (Hg.) 1999, S. 177-213.

Goodin, R. E. (1996), Institutionalizing the Public Interest: The Defense of Deadlock and Beyond, in: American Political Science Review 90/2, S. 331-343.

Greven, M. Th. (Hg., 1998), Demokratie - eine Kultur des Westens? 20. Wissenschaftlicher Kongreß der Deutschen Vereinigung für Politische Wissenschaft, Opladen.

Greven, M. Th. (1998a), Mitgliedschaft, Grenzen und politischer Raum: Problemdimensionen der Demokratisierung in der Europäischen Union, in: B. Kohler-Koch (Hg.) 1998, S. 249-270.

Greven, M. Th./Schmalz-Bruns, R. (Hg., 1999), Politische Theorie - heute. Ansätze und Perspektiven, Baden-Baden.

Gunn, J. A. W. (1989), Public Interest, in: Political Innovation and Conceptual Change, hg. v. T. Ball, J. Farr u. R.L. Hanson, Cambridge, S. 194-210.

Gutmann, A./Thompson, D. (1996), Democracy and Disagreement. Why moral conflict cannot be avoided in politics, and what should be done about it, Cambridge/Mass.

Habermas, J. (1996), Die Einbeziehung des Anderen, Frankfurt/M.

Habermas, J. (1996a), Der europäische Nationalstaat - Zur Vergangenheit und Zukunft von Souveränität und Staatsbürgerschaft, in: J. Habermas 1996, S. 128-153.

Habermas, J. (1996b), Replik auf Beiträge zu einem Symposion der Cardozo Law School, in: J. Habermas 1996, S. 309-398.

Habermas, J. (1996c), Braucht Europa eine Verfassung? Eine Bemerkung zu Dieter Grimm, in: J. Habermas 1996, S. 185-191.

Habermas, J. (1996d), Inklusion - Einbeziehen oder Einschließen? Zum Verhältnis von Nation, Rechtsstaat und Demokratie, in: J. Habermas 1996, S. 154-184.

Habermas, J. (1998), Die postnationale Konstellation und die Zukunft der Demokratie, in: J. Habermas 1998, S. 91-169.

Hibst, P. (1990), Gemeiner Nutzen. Begriffsgeschichtliche Untersuchungen zur politischen Theorie vom 5. vorchristlichen bis zum 15. nachchristlichen Jahrhundert, in: Archiv für Begriffsgeschichte 33, S. 60-95.

Honneth, A. (1992), Kampf um Anerkennung. Zur moralischen Grammatik sozialer Konflikte, Frankfurt/M.

Honneth, A. (1999), Demokratie als reflexive Kooperation. John Dewey und die Demokratietheorie der Gegenwart, in: H. Brunkhorst/P. Niesen (Hg.) 1999, S. 37-65.

Hurka, Th. (1997), The Justification of National Partiality, in: R. McKim/J. McMahan (Hg.) 1997, S. 139-157.

Joas, H. (1997), Die Entstehung der Werte, Frankfurt/M.

Johnson, J. (1998), Arguing for Deliberation: Some Skeptical Considerations, in: J. Elster (Hg.) 1998, S. 161-184.

Kempshall, M. S. (1999), The Common Good in Late Medieval Political Thought, Oxford.

Kersting, W. (1998). Einleitung: Probleme der politischen Philosophie der internationalen Beziehungen: die Beiträge im Kontext, in: Ch. Chwaszca/W. Kersting (Hg.) 1998, S. 9-69.

Kettner, M. (1998), John Deweys demokratische Experimentiergemeinschaft, in: H. Brunkhorst (Hg.) 1998 , S. 44-66. 
Knight, J./Johnson, J. (1996), Political Consequences of Pragmatism, in: Political Theory 24/1, S. 68-96.

Knight, J./Johnson, J. (1999), Inquiry into Democracy: What Might a Pragmatist Make of Rational Choice Theories?, in: American Journal of Political Science 43/2, S. 566-589.

Kohler-Koch, B. (Hg., 1998), Regieren in entgrenzten Räumen (= PVS Sonderheft 29). Opladen.

Luhmann, N. (1998), Die Politik der Gesellschaft, Bielefeld.

MacGilvray, E. A. (1999), Experience as Experiment: Some Consequences of Pragmatism for Democratic Theory, in: American Journal of Political Science 43/2, S. 542-565.

March, J. G./Olsen, J. P. (1995), Democratic Governance, New York.

Mattern, M. (1999), John Dewey, Art and Public Life, in: The Journal of Politics 61/1, S. 54-75.

McKim, R./McMahan, J. (Hg., 1997), The Morality of Nationalism. Oxford/New York.

McMahan, J. (1997), The Limits of National Partiality, in: R. McKim/J. McMahan (Hg.) 1997, S. 107-138.

Miller, D. (1995), On Nationality. Oxford.

Morris, D. (1999), „How Shall We Read What We Call Reality?“: John Dewey’s New Science of Democracy, in: American Journal of Political Science 43/2, S. 608-628.

Münkler, H. (1991), Die Idee der Tugend. Ein politischer Leitbegriff im vorrevolutionären Europa, in: Archiv für Kulturgeschichte 73, S. 379-403.

Münkler, H./Fischer, K. (1999), Gemeinwohl und Gemeinsinn. Thematisierung und Verbrauch soziomoralischer Ressourcen in der modernen Gesellschaft, in: Berlin-Brandenburgische Akademie der Wissenschaften: Berichte und Abhandlungen, Bd. 7, Berlin, S. 237-265.

O'Neill, O. (1998a), Justice and Boundaries, in: Ch. Chwaszca/W. Kersting (Hg.) 1998, S. 502-520.

O'Neill, O. (1998b), Transnationale Gerechtigkeit, in: Philosophie der Menschenrechte, hg. v. St. Gosepath u. G. Lohmann, Frankfurt/M., S. 188-232.

Offe, C. (1986), Die Utopie der Null-Option. Modernität und Modernisierung als politische Gütekriterien, in: Moderne oder Postmoderne?, hg. v. P. Koslowski, R. Spaemann u. R. Löw, Weinheim, S. $143-172$.

Offe, C. (1997), Micro-aspects of democratic theory: what makes for the deliberative competence of citizens, in: Democracy's Victory and Crisis, hg. v. A. Hadenius, Cambridge, S. 81-104.

Offe, C. (1998a), „Homogeneity“ and Constitutional Democracy: Coping with Identity Conflicts through Group Rights, in: The Journal of Political Philosophy 6/2, S. 113-141.

Offe, C. (1998b), Demokratie und Wohlfahrtsstaat: Eine europäische Regimeform unter dem Streß der europäischen Integration, in: W. Streeck (Hg.) 1998, S. 99-136

Offe, C. (1999), How can we trust our fellow citizens? In: Democracy \& Trust, hg. v. M. Warren, Cambridge, S. 42-87 (dt.: Wie können wir unseren Mitbürgern vertrauen? In: Vertrauen. Die Grundlagen des sozialen Zusammenhalts, hg. v. M. Hartmann u. C. Offe, Frankfurt/M. 2001, S. 241-294).

Przeworski, A. (1998), Deliberation and Ideological Domination, in: J. Elster (Hg.) 1998, S. 140-160.

Putnam, H. (1997), Deweys Politikbegriff - eine Neubewertung, in: Ders., Für eine Erneuerung der Philosophie, Stuttgart, S. 227-252.

Rawls, J. (1979), Eine Theorie der Gerechtigkeit, Frankfurt/M.

Ripstein, A. (1997), Context, Continuity, and Fairness, in: R. McKim/J. McMahan (Hg.) 1997, S. 209-226.

Sanders, L. M. (1997), Against Deliberation, in: Political Theory 25/3, S. 347-376. 
Saretzki, Th. (1996a), Wie unterscheiden sich Argumentieren und Verhandeln? Definitionsprobleme, funktionale Bezüge und strukturelle Differenzen von zwei Kommunikationsmodi, in: Verhandeln und Argumentieren. Dialog, Interessen und Macht in der Umweltpolitik, hg. v. V. v. Prittwitz, Opladen, S. 19-39.

Saretzki, Th. (1996b), Verhandelte Diskurse? Probleme der Vermittlung von Argumentation und Partizipation am Beispiel des TA-Verfahrens zum „Anbau gentechnisch erzeugter Herbizidresistenz" am Wissenschaftszentrum Berlin. In: Verhandeln und Argumentieren. Dialog, Interessen und Macht in der Umweltpolitik, hg. v. V. v. Prittwitz, Opladen, S. 135-167.

Saretzki, Th. (1999a), Post-Positivistische Policy-Analyse und deliberative Demokratie, in: Bürgersinn und Kritik. Festschrift für U. Bermbach zum 60. Geburtstag, hg. v. M. Th. Greven, H. Münkler u. R. Schmalz-Bruns, Baden-Baden, S. 297-321.

Saretzki, Th. (1999b), Technologische Bürgerschaft? Anmerkungen zur Konstruktion von „citizenship“ in einer technologischen „polity“, in: Demokratie und Technik, hg. v. R. Martinsen u. G. Simonis, Opladen.

Scheffler, S. (1997), Liberalism, Nationalism, and Egalitarianism, in: R. McKim/J. McMahan (Hg.), S. 191-208.

Schmalz-Bruns, R. (1995), Reflexive Demokratie. Die demokratische Transformation moderner Politik, Baden-Baden.

Schmalz-Bruns, R. (1998a), Grenzerfahrungen und Grenzüberschreitungen: Demokratie im integrierten Europa?, in: B. Kohler-Koch (Hg.), S. 369-380.

Schmalz-Bruns, R. (1998b), Kunst und Demokratie. Modelle ästhetischer Gesellschaftskritik und Politik, in: Bürgersinn und Kritik. Festschrift für Udo Bermbach zum 60. Geburtstag, hg. v. M. Th. Greven, H. Münkler u. R. Schmalz-Bruns, Baden-Baden, S. 413-441.

Smiley, M. (1990), Pragmatic Inquiry and Social Conflict: A Critical Reconstruction of Dewey's Model of Democracy, in: Praxis International 9/4, S. 365-380.

Smiley, M. (1999), Pragmatic Inquiry and Democratic Politics. In: American Journal of Political Science 43/2, S. 629-647.

Stokes, S. (1998), Pathologies of Deliberation, in: Jon Elster (Hg.) 1998, S. 123-139.

Stolleis, M. (1995), „Staatsethik“, oder: Vom sittlichen Staat zu den Bürgertugenden, in: Kritische Vierteljahresschrift für Gesetzgebung und Rechtswissenschaft 78/1, S. 58-68.

Streeck, W. (Hg., 1998), Internationale Wirtschaft, nationale Demokratie. Herausforderungen für die Demokratietheorie. Frankfurt/M./New York.

Streeck, W. (1998a), Einleitung: Internationale Wirtschaft, nationale Demokratie?, in: W. Streeck (Hg.) 1998, S. 11-58.

Van den Daele, W./Neidhardt, F. (1996) „Regieren durch Diskussion“ - Über Versuche, mit Argumenten Politik zu machen, in: Kommunikation und Entscheidung. Politische Funktionen öffentlicher Meinungsbildung und diskursiver Verfahren, hg. v. W. van den Daele u. F. Neidhardt, Berlin, S. 9-50.

Wingert, L. (1993), Gemeinsinn und Moral. Grundzüge einer intersubjektivistischen Moralkonzeption, Frankfurt/M.

Wirsching, A. (1990), Bürgertugend und Gemeininteresse. Zum Topos der „Mittelklassen“ in England im späten 18. und frühen 19. Jahrhundert, in: Archiv für Kulturgeschichte 72/1, S. 173-199.

Young, I. M. (1996), Communication and the Other: Beyond Deliberative Democracy, in: Democracy and Difference. Contesting the Boundaries of the Political, hg. v. S. Benhabib, Princeton, NJ, S. 120-135. 
